\title{
Genetic Analysis of a Cohort of 275 Patients with Hyper-lgE Syndromes and/or Chronic Mucocutaneous Candidiasis
}

\author{
Natalie Frede ${ }^{1,2}$. Jessica Rojas-Restrepo ${ }^{1,3}$. Andrés Caballero Garcia de Oteyza ${ }^{1,3} \cdot$ Mary Buchta ${ }^{1}$. \\ Katrin Hübscher ${ }^{1,3}$. Laura Gámez-Díaz ${ }^{1,3}$. Michele Proietti ${ }^{1,3}$. Shiva Saghafi ${ }^{4} \cdot$ Zahra Chavoshzadeh $^{5}$. \\ Pere Soler-Palacin ${ }^{6} \cdot$ Nermeen Galal $^{7} \cdot$ Mehdi Adeli $^{8}$. Juan Carlos Aldave-Becerra ${ }^{9} \cdot$ Moudjahed Saleh Al-Ddafari $^{10}$. \\ Ömür Ardenyz $z^{11} \cdot$ T. Prescott Atkinson ${ }^{12} \cdot$ Fulya Bektas Kut $^{13} \cdot$ Fatih Çelmeli $^{14} \cdot$ Helen Rees $^{15} \cdot$ Sara S. Kilic $^{16}$. \\ Ilija Kirovski ${ }^{17}$. Christoph Klein ${ }^{18}$ - Robin Kobbe ${ }^{19}$. Anne-Sophie Korganow ${ }^{20}$. Desa Lilic ${ }^{21}$ - Peter Lunt L $^{22}$. \\ Niten Makwana ${ }^{23}$. Ayse Metin ${ }^{24} \cdot$ Tuba Turul Özgür $^{25}$. Ayse Akman Karakas ${ }^{26}$. Suranjith Seneviratne ${ }^{27}$. \\ Roya Sherkat ${ }^{28}$. Ana Berta Sousa ${ }^{29}$. Ekrem Unal ${ }^{30,31}$. Turkan Patiroglu ${ }^{32} \cdot$ Volker Wahn $^{33}$. \\ Horst von Bernuth ${ }^{33,34,35,36} \cdot$ Margo Whiteford $^{37}$ - Rainer Doffinger ${ }^{38} \cdot$ Zineb Jouhadi $^{39}$ - Bodo Grimbacher 1,3,40,41,42,43
}

Received: 11 August 2020 / Accepted: 5 June 2021 / Published online: 14 August 2021

(c) The Author(s) 2021

\begin{abstract}
Hyper-IgE syndromes and chronic mucocutaneous candidiasis constitute rare primary immunodeficiency syndromes with an overlapping clinical phenotype. In recent years, a growing number of underlying genetic defects have been identified. To characterize the underlying genetic defects in a large international cohort of 275 patients, of whom 211 had been clinically diagnosed with hyper-IgE syndrome and 64 with chronic mucocutaneous candidiasis, targeted panel sequencing was performed, relying on Agilent HaloPlex and Illumina MiSeq technologies. The targeted panel sequencing approach allowed us to identify 87 (32 novel and 55 previously described) mutations in 78 patients, which generated a diagnostic success rate of 28.4\%. Specifically, mutations in DOCK8 (26 patients), STAT3 (21), STAT1 (15), CARD9 (6), AIRE (3), ILI7RA (2), SPINK5 (3), ZNF341 (2), CARMIL2/RLTPR (1), IL12RB1 (1), and WAS (1) have been detected. The most common clinical findings in this cohort were elevated IgE (81.5\%), eczema (71.7\%), and eosinophilia (62.9\%). Regarding infections, $54.7 \%$ of patients had a history of radiologically proven pneumonia, and $28.3 \%$ have had other serious infections. History of fungal infection was noted in $53 \%$ of cases and skin abscesses in 52.9\%. Skeletal or dental abnormalities were observed in $46.2 \%$ of patients with a characteristic face being the most commonly reported feature $(23.1 \%)$, followed by retained primary teeth in $18.9 \%$ of patients. Targeted panel sequencing provides a cost-effective first-line genetic screening method which allows for the identification of mutations also in patients with atypical clinical presentations and should be routinely implemented in referral centers.
\end{abstract}

Keywords Primary immunodeficiency $\cdot$ Hyper-IgE syndrome $\cdot$ Chronic mucocutaneous candidiasis $\cdot$ Genetics $\cdot$ Targeted panel sequencing $\cdot$ Next-generation sequencing

\section{Introduction}

Hyper-IgE syndromes (HIES) and chronic mucocutaneous candidiasis (CMC) constitute rare primary immunodeficiency syndromes with overlapping phenotypes. HIES have traditionally been characterized by the clinical triad

Bodo Grimbacher

bodo.grimbacher@uniklinik-freiburg.de

Extended author information available on the last page of the article of recurrent pneumonias, recurrent skin abscesses, and markedly elevated serum IgE levels [1-3]. Eczema and eosinophilia represent further hallmarks. The most common underlying genetic defects are loss of function mutations of the transcription factor STAT3 [4, 5], which, in addition to the triad already mentioned, are associated with dental, skeletal, and connective tissue abnormalities $[4,5]$. In contrast, $D O C K 8$ deficiency is characterized by severe viral infections, e.g., with herpes viruses, papilloma viruses, and molluscum contagiosum virus as well as the increased 
occurrence of malignancies, especially hematological and epithelial cancers. DOCK 8 deficiency is classified as a combined immunodeficiency in the latest International Union of Immunological Societies (IUIS) classification [6, 7]. More recently, mutations in CARD11, ERBB2IP, IL6R, IL6ST, $P G M 3, T G F B R 1 / 2$, and ZNF431 have been recognized as further genetic causes of HIES-like phenotypes [8-11]. A similar phenotype may be produced by genetic skin disorders such as Comèl-Netherton syndrome, an ichthyosis syndrome caused by mutations in SPINK5 encoding, a serine protease essential for skin barrier integrity. Also, severe atopic dermatitis (AD) may lead to a similar phenotype, which may be hard to distinguish from primary immunodeficiencies as the impaired barrier function in AD may lead to infections. The hyper-IgE phenotype overlaps with the one of chronic mucocutaneous candidiasis (CMC) in that patients may show increased susceptibility to fungal infections. CMC is characterized by enhanced susceptibility to infections caused by Candida ssp. and dermatophytes [12]. While the majority of patients suffer from recurrent skin, nail, or mucous membrane infections, a smaller subgroup of patients develops invasive fungal disease associated with a high burden of morbidity and mortality. In recent years, a growing number of genetic defects underlying CMC have been identified, which confirm a role for both the innate and the adaptive immune systems (CARD9, STAT1, ACT1, IL-17F, IL-17RA, IL17RC, AIRE, IL12B, IL12RB1, RORC) in antifungal immunity [13-16]. Depending on the underlying molecular defect, additional clinical manifestations may include autoimmunity, endocrinopathy, increased susceptibility to bacterial infections, and malignancies. Laboratory findings may also include elevated $\operatorname{IgE}$ and eosinophilia, adding to a common hyper-IgE phenotype.

With a rising number of known underlying genetic defects and overlapping clinical phenotypes, novel sequencing techniques have been gaining importance to reach a definite diagnosis. Obtaining a genetic diagnosis in these patients with inborn errors of immunity is crucial for identifying the best course of treatment and counseling patients and their families regarding the prognosis and further family planning. The advent of next-generation sequencing (NGS) has since greatly facilitated this process at reduced costs and a shorter turnaround time by allowing the simultaneous analysis of a multitude of genes. In order to promote the time- and cost-effective identification of the genetic diagnosis, we have established a targeted panel sequencing approach relying on Agilent HaloPlex and Illumina MiSeq technologies. Here, we present our results on targeted panel sequencing of known disease-causing genes in a cohort of 275 patients with a clinical diagnosis of HIES or CMC. This approach allowed us to identify 87 mutations in 78 patients.

\section{Methods}

\section{Patients}

This study was conducted under the ethics protocols 239/99-120,733 and 302/13 (ethics committee of the University Hospital of Freiburg, Germany). All patients, or for children their legal guardians, have consented according to local ethics guidelines. DNA or whole blood samples of patients who had either a clinical diagnosis of HIES or CMC, and whose samples were referred to our laboratory for genetic workup on a research basis by their local physicians, were obtained. Around 211 patients had a clinical diagnosis of HIES, while 64 patients were diagnosed with CMC. With two exceptions, all patients were unrelated index patients. If DNA samples were available, other family members were subsequently investigated for the detected mutations by Sanger sequencing. The investigated patients were from Algeria (5 patients), Belgium (2), Bosnia (1), Brazil (1), Canada (2), Chile (2), Colombia (2), Denmark (1), Egypt (30), Finland (1), France (3), Germany (41), Great Britain (44), Greece (3), India (2), Iran (34), Ireland (8), Israel (5), Italy (10), Lebanon (1), Libya (2), Macedonia (2), Malaysia (1), Morocco (21), Oman (1), Peru (3), Portugal (3), Qatar (1), Slovakia (1), Spain (3), Switzerland (2), Tunisia (4), Turkey (29), USA (3), and the West Indies (1). The cohort comprises an initial group of 90 patients, in whom conventional diagnostics including Sanger sequencing of the suspected underlying target gene (STAT3, DOCK8, PGM3, STAT1) had not led to a definite molecular diagnosis. Subsequently, the cohort was expanded to include patients with a clinical diagnosis of HIES or CMC without prior Sanger sequencing.

\section{DNA Extraction}

DNA extraction was performed according to local protocols. In brief, erythrocytes were lysed with RBC buffer, and the remaining nucleated cells were subjected to Qiagen Cell Lysis Solution. Qiagen Protein Precipitation Solution was used to precipitate the proteins. The DNA was subsequently precipitated with isopropanol, washed with ethanol, and resuspended and stored in Qiagen DNA Hydration Solution.

\section{Panel Design}

A customized gene panel was designed through Agilent's web-based SureDesign application. The panel design initially comprised 15 genes in which published evidence has 
established that mutations can cause HIES or CMC and was updated regularly to optimize coverage and include new candidate genes. A list of sequenced gene sets is found in Supplemental Table 1.

\section{Target Enrichment and Sequencing}

Targeted enrichment was performed with Agilent's HaloPlex Target Enrichment System for Illumina sequencing. The manufacturer's instructions as detailed in Agilent's user manual were followed. In brief, DNA samples were subjected to digestion by adding a restriction enzyme master mix prepared following the kit's protocol and an incubation step at $37^{\circ} \mathrm{C}$, and digestion was validated by gel electrophoresis. Subsequently, the restriction fragments were hybridized to the HaloPlex probe capture library by the addition of a hybridization master mix and indexing primer cassettes. The mix was incubated at $95^{\circ} \mathrm{C}$ for $10 \mathrm{~min}$ followed by a 3-h incubation at $54{ }^{\circ} \mathrm{C}$. The target DNA was captured with a biotin-streptavidin system using HaloPlex Magnetic Beads. After a washing step, the circular fragments were closed through a ligation reaction at $55^{\circ}$. The captured target libraries were amplified by PCR with the master mix prepared according to the manufacturer's instructions. In a final step, the amplified target libraries were purified through AMPure XP beads and washed in ethanol. Enrichment was validated on an Agilent TapeStation system.

Samples were pooled in equimolar amounts for multiplex sequencing on an Illumina MiSeq system. An Illumina v2 reagent kit was used, and the provided protocol was followed. Libraries were denatured with $\mathrm{NaOH}$ and diluted to a final concentration of 8-12 pM. For loading and starting the MiSeq system, the manufacturer's instructions as detailed in the MiSeq System User Guide were followed.

\section{Data Analysis}

Data analysis was performed using Agilent's SureCall software. In brief, this included the trimming of adapter sequences and alignment to the human reference genome (hg19/GRCh37) using Burrows-Wheeler aligner. The Agilent SNP caller was used for detecting single nucleotide variants. SNP filtering, mutation classification, and annotation were performed as part of the SureCall analysis workflow. Copy number variants were determined from sequencing coverage data using a genomic analysis toolkit. Single nucleotide variants with an allele frequency $>0.01$ in the general population were excluded. Detected missense, nonsense, frameshift, and splice site mutations were annotated. For prioritization databases, such as HGMD (Human Gene Mutation Database) [17], ExAc (Exome Aggregation Consortium) [18], and dbSNP (the Single Nucleotide Polymorphism Database) [19], as well as polymorphism phenotyping tools/mutation severity predictors such as PolyPhen2 [20] and combined annotation-dependent depletion (CADD) [21] were used.

\section{Quality Control Data}

The average read depth of all detected mutations was $914 \times$. Mutations were confirmed by Sanger sequencing or PCR amplification in the case of larger deletions. Around 91.8\% of all bases in target regions were covered at least 100-fold, and $97.6 \%$ were covered at least 20 -fold.

\section{Sanger Sequencing}

All detected mutations were confirmed by Sanger sequencing according to established protocols [22]. Regions of interest were amplified from genomic DNA by standard PCR. PCR primers were used for Sanger sequencing according to standard techniques. All primer sequences are available upon request. Sequencing was performed on an AB3130x1 Genetic Analyzer, or PCR products were sent for commercial sequencing at GATC (Konstanz, Germany).

\section{PCR}

In order to confirm suspected copy number variations (deletions), the regions in question were amplified from genomic DNA by PCR as detailed previously. Amplification of patient and control DNA was checked by agarose gel electrophoresis.

\section{Flow Cytometry}

If available, functional testing on patient cells was performed for novel variants. While not diagnostic, phosphorylation assays contribute to the assessment of potential pathogenicity and mechanism of STAT variants. Cell surface staining or intracellular staining for the assessment of variants was performed by flow cytometry. PBMCs from patients were stained with pPSTAT3-PE, pSTAT1-Alexa 647, or IL17RAAPC and were subsequently analyzed on a BD FACS Canto II.

\section{Results}

Within this study, we analyzed a cohort of 275 patients by targeted panel sequencing, out of whom 211 had been clinically diagnosed with HIES by their referring physicians and 64 with CMC. Targeted panel sequencing identified underlying disease-causing mutations in 78 patients, i.e., $28.4 \%$ of all analyzed patients. Specifically, we detected 25 homozygous and one compound heterozygous mutations in DOCK8, 
21 heterozygous STAT3 mutations, 13 heterozygous mutations in STATl, five homozygous and one compound heterozygous mutation in $C A R D 9$, two compound heterozygous and one heterozygous mutation in AIRE, three homozygous mutations in SPINK5, one homozygous and one compound heterozygous mutation in IL17RA, two homozygous ZNF34I mutations, one homozygous RLTPR mutation, one homozygous mutation in ILI2RBI, and one hemizygous WAS mutation in this cohort (Fig. 1). The average coverage of detected mutations in this study amounted to 914 reads.

The mean age of patients at the time point of sequencing was 17.9 years (range: 1-62 years; mean for patients with clinical hyper-IgE phenotype: 15.7 years, for patients with CMC: 24.3 years). Around $55.1 \%$ of analyzed patients were male and $44.9 \%$ female. Around $20.5 \%$ were reported to be consanguineous. The mean age at first clinical presentation was 8.41 years (median: 5 years, range: $0-51$ years).

Clinical data were available for 183 patients and are summarized in Fig. 2. The majority of patients had elevated IgE levels $(81.5 \%)$, and $62.9 \%$ had eosinophilia. The most commonly reported symptom was eczema (71.7\%). Regarding infections, $54.7 \%$ of patients had a history of radiologically proven pneumonia, while $28.3 \%$ of patients were reported to have had other serious infections. Twelve patients had passed away from infectious causes. Around 53\% of patients in this cohort had a history of mucocutaneous candidiasis or fungal infection and 52.9\% had a history of skin abscesses. About $37.2 \%$ had recurrent sinusitis with at least 3 episodes per year. Around $46.2 \%$ of patients had skeletal or dental abnormalities with a characteristic face being the most commonly reported item (23.1\%), followed by retained primary teeth reported in $18.9 \%$ of patients. Around 9.8\% had hyperextensibility and $8.5 \%$ a history of fractures with inadequate trauma.

Sixteen patients were reported to have enteropathy. Neurologic features were generally rare (reported in 7 patients) and included epilepsy, delayed development, encephalomalacia, and cerebral atrophy. Malignancy or suspicion thereof was only reported in three patients, one of which had a sarcoma, one multiple skin tumors, and another a lymphoma. HIES patients had an average NIH HIES score of 34.0 points $(n=122$; min.: 6, max.: 73), whereas CMC patients scored an average of 9.75 points $(n=20)$.

\section{Identified Genetic Defects in Patients with a Clinical Diagnosis of HIES}

Out of 211 patients referred for genetic workup with a clinical diagnosis of HIES, we were able to make a genetic diagnosis in 54 patients, i.e., 25.6\% . A detailed list of detected mutations and clinical presentations of patients can be found in Table 1. In this cohort, DOCK8 mutations were the most common underlying genetic defect. A total of 26 patients were shown to have homozygous or compound heterozygous DOCK 8 mutations. Out of these, four patients had splice site defects, six nonsense mutations, and five frameshift mutations. One patient had a one amino acid deletion, whereas another patient (HIES53) had a compound heterozygous mutation consisting of a heterozygous one amino acid deletion and a heterozygous splice site variant. Staining for

\section{HIES}
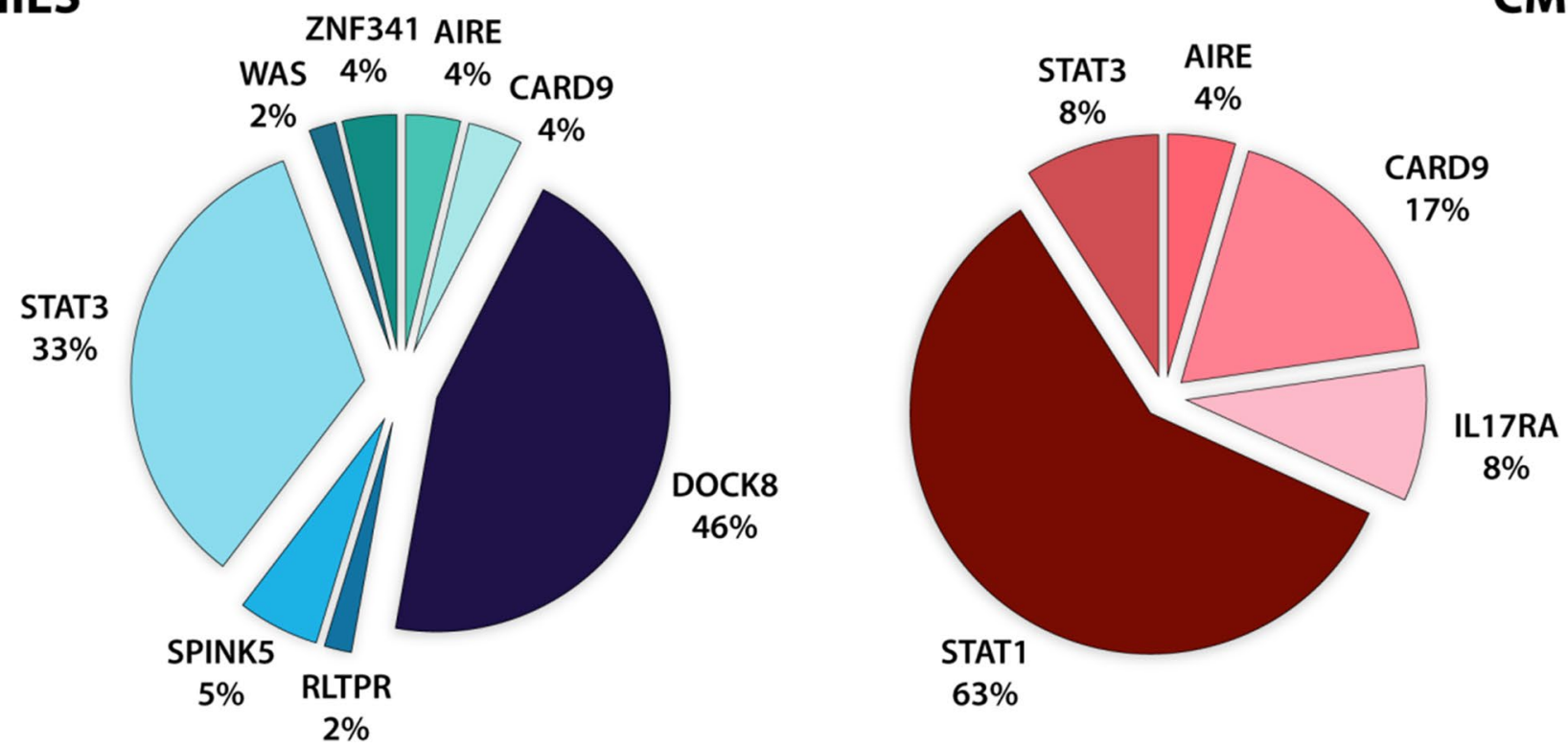

Fig. 1 Frequency of genetic defects in patients with a clinical diagnosis of HIES and CMC: In patients with a clinical diagnosis of HIES, DOCK8 and STAT3 mutations constituted the most common defects, whereas in CMC patients, STAT1 mutations were the most common defects 
Fig. 2 Clinical patient characteristics: heat map showing relative scoring for presence and severity of clinical patient characteristics in this cohort. Eosinophilia, elevated IgE, and eczema cluster together

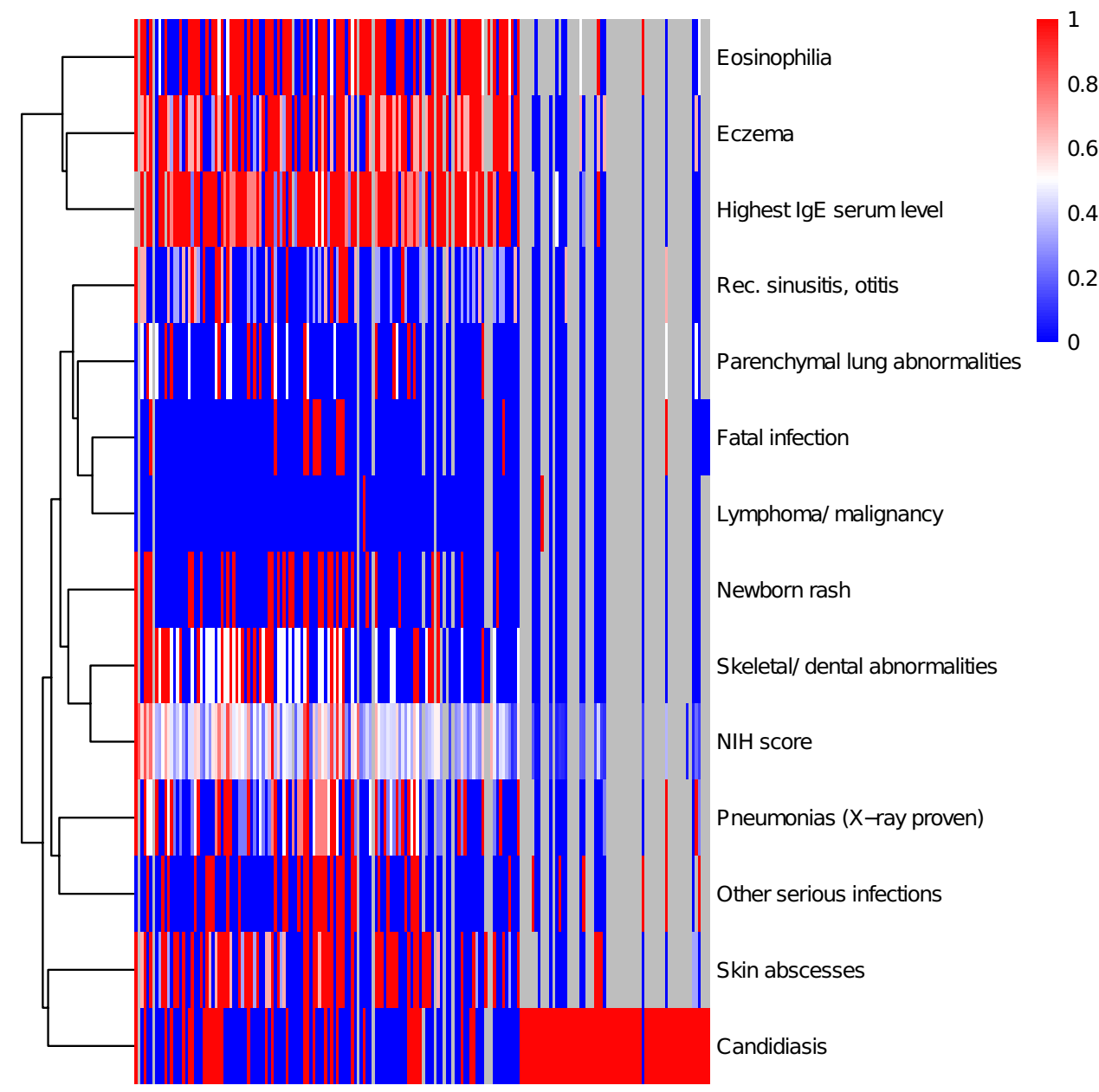

DOCK8 protein confirmed severely reduced protein expression by flow cytometry. Seven patients were found to have larger homozygous exonic deletions, which could be confirmed by PCR amplification.HIES46

Eighteen patients had heterozygous STAT3 mutations. Of these, two patients had splice site mutations, one a nonsense mutation, two single amino acid deletions, and 13 patients had missense mutations. Functional testing was performed for the novel variants p.K531Q and p.I711F as well as for patient HIES46, who had two previously undescribed missense variants, p.L571F and p.Y575D. Testing showed reduced STAT3 phosphorylation consistent with a loss of function for two patients and mildly reduced phosphorylation of unclear significance for the third patient (Fig. 3). Furthermore, we identified two patients with a homozygous ZNF341 nonsense mutation [23]. Biallelic mutations in ZNF341 lead to diminished STAT3 expression, resulting in hyper-IgE syndrome [23, 24]. In addition to recurrent respiratory infections, skin abscesses, and severe eczema, the two patients suffered from micrognathia.

Interestingly, two patients clinically diagnosed with HIES were shown to have previously published CARD9 mutations (p.R373P and p.Q295*) associated with chronic mucocutaneous candidiasis, respectively, deep dermatophytosis. Patient HIES03 had IgE levels of > 20,000 IU/1 and marked eosinophilia adding towards a total NIH HIES score of 40 points [25]. However, he had suffered from candida meningitis, and thus invasive fungal infection, hinting towards a $C A R D 9$ defect. Notably, no clinical sign or history of lung disease - typical of HIES - was present. Patient HIES04 suffered from persistent thrush in infancy and candida nail infection; however, he had been diagnosed with HIES due to elevated IgE and hypereosinophilia.

Three patients clinically diagnosed with HIES had homozygous mutations in SPINK5, which cause Comèl-Netherton syndrome, a rare syndrome associated with erythroderma, ichthyosis, short and brittle "bamboo" hair, atopy, and elevated IgE. One patient had a nonsense mutation, one a frameshift, and one a splice site mutation. All patients had severe eczema and elevated IgE, and only one of the three patients did not have a history of recurrent infections.

Notably, another patient (HIES50) clinically diagnosed with HIES was shown to have a hemizygous mutation in WAS (p.P403L), which had previously been published as causing mild immunodeficiency, muscle dystonia, and 


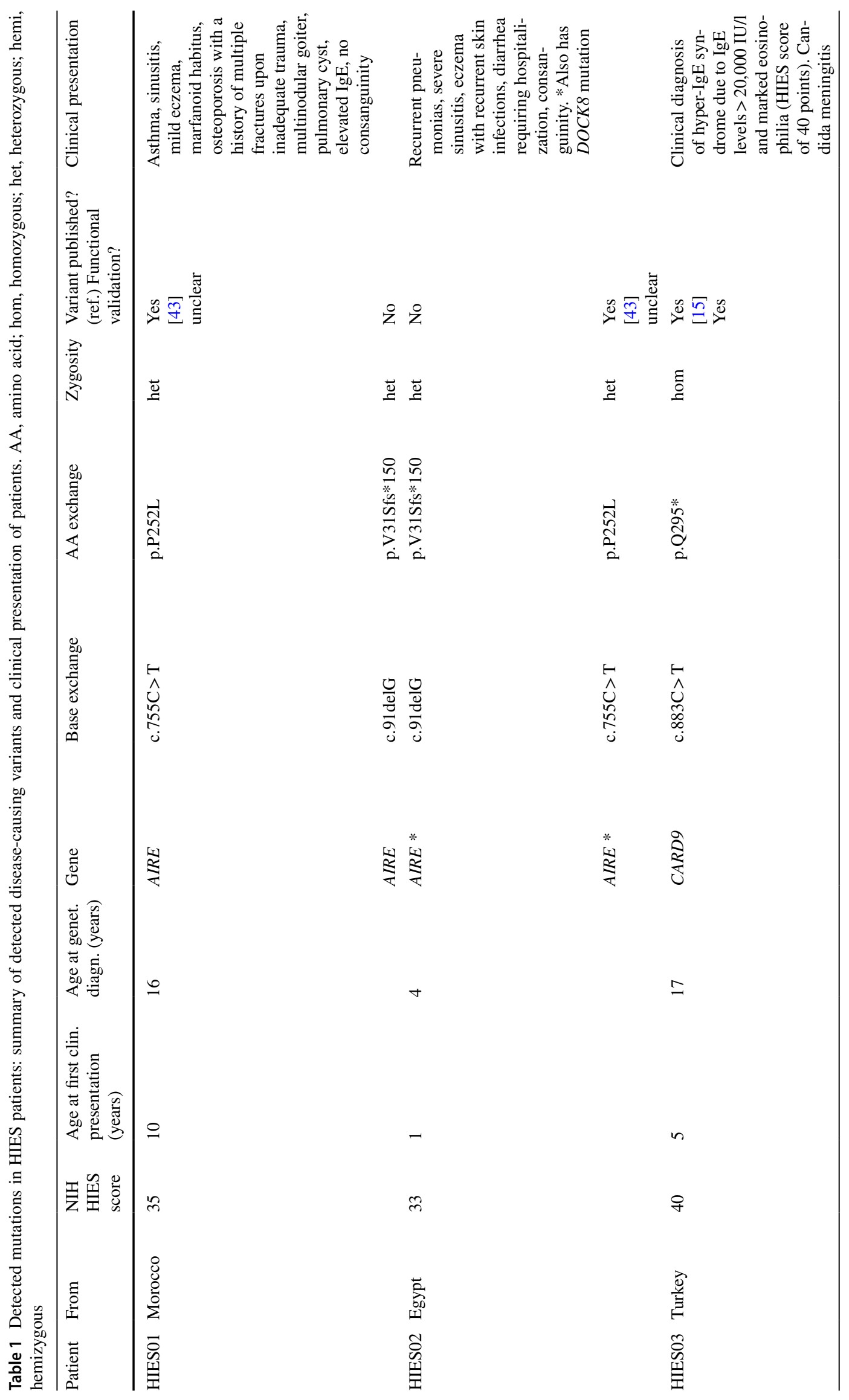




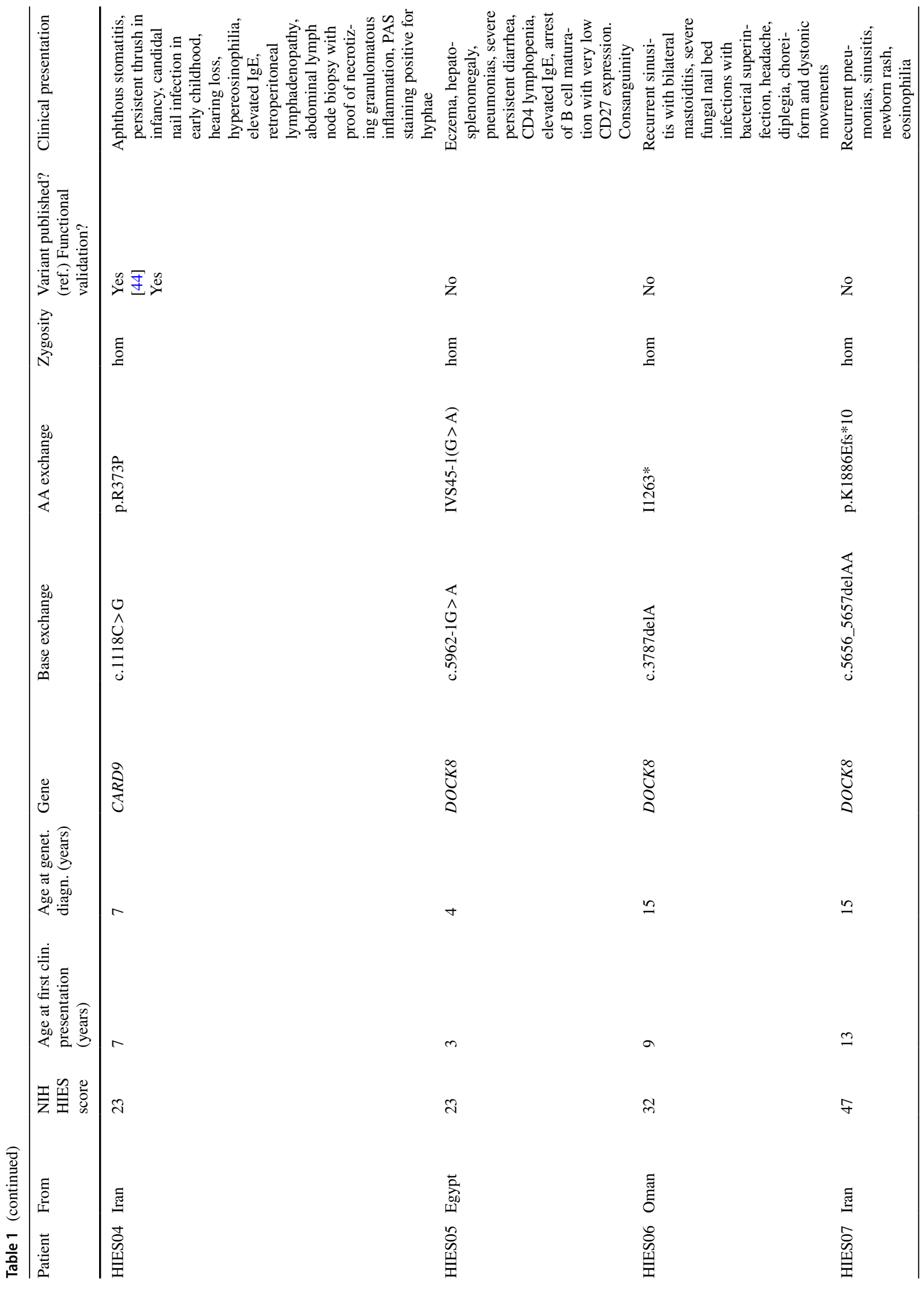




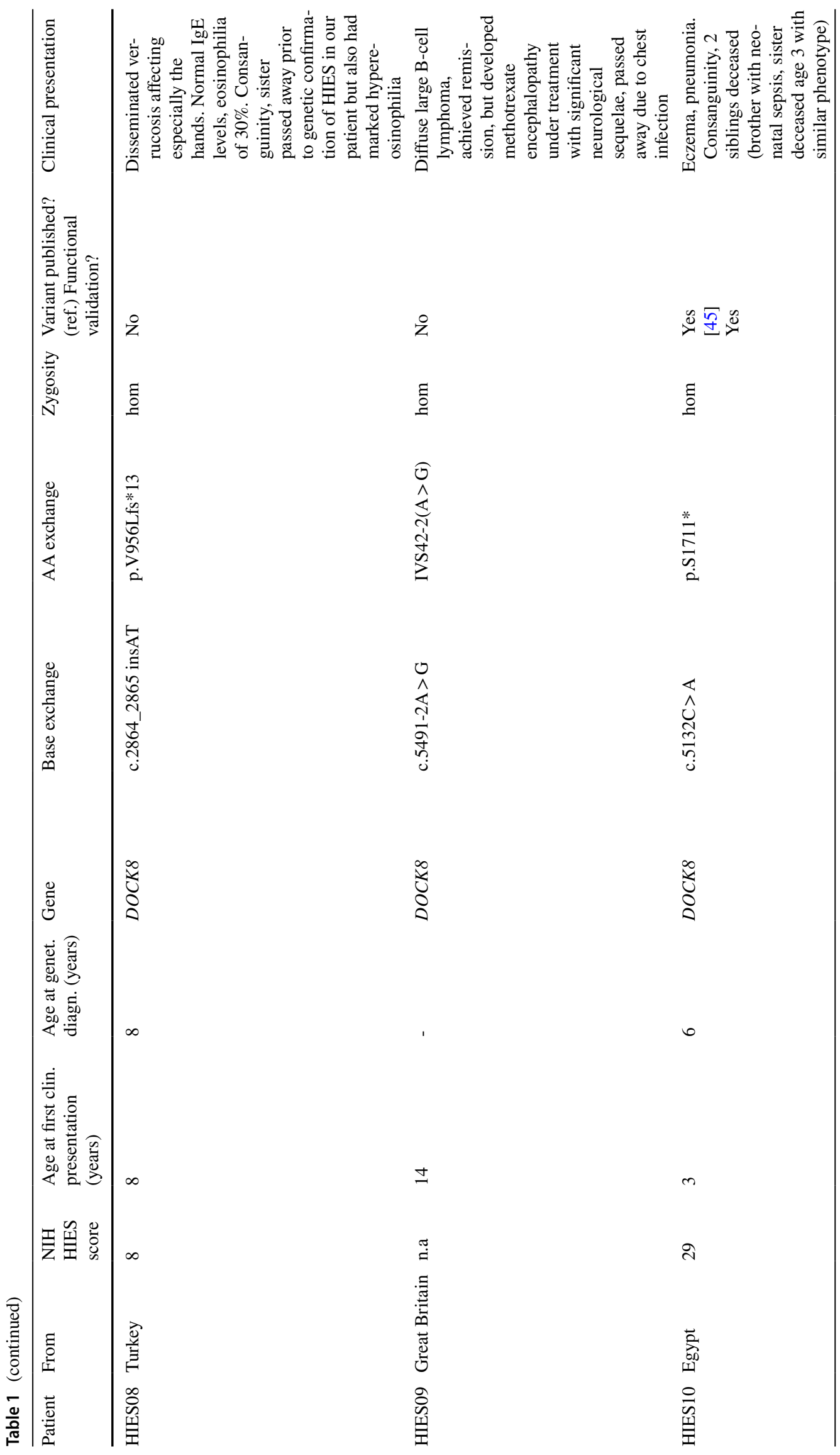




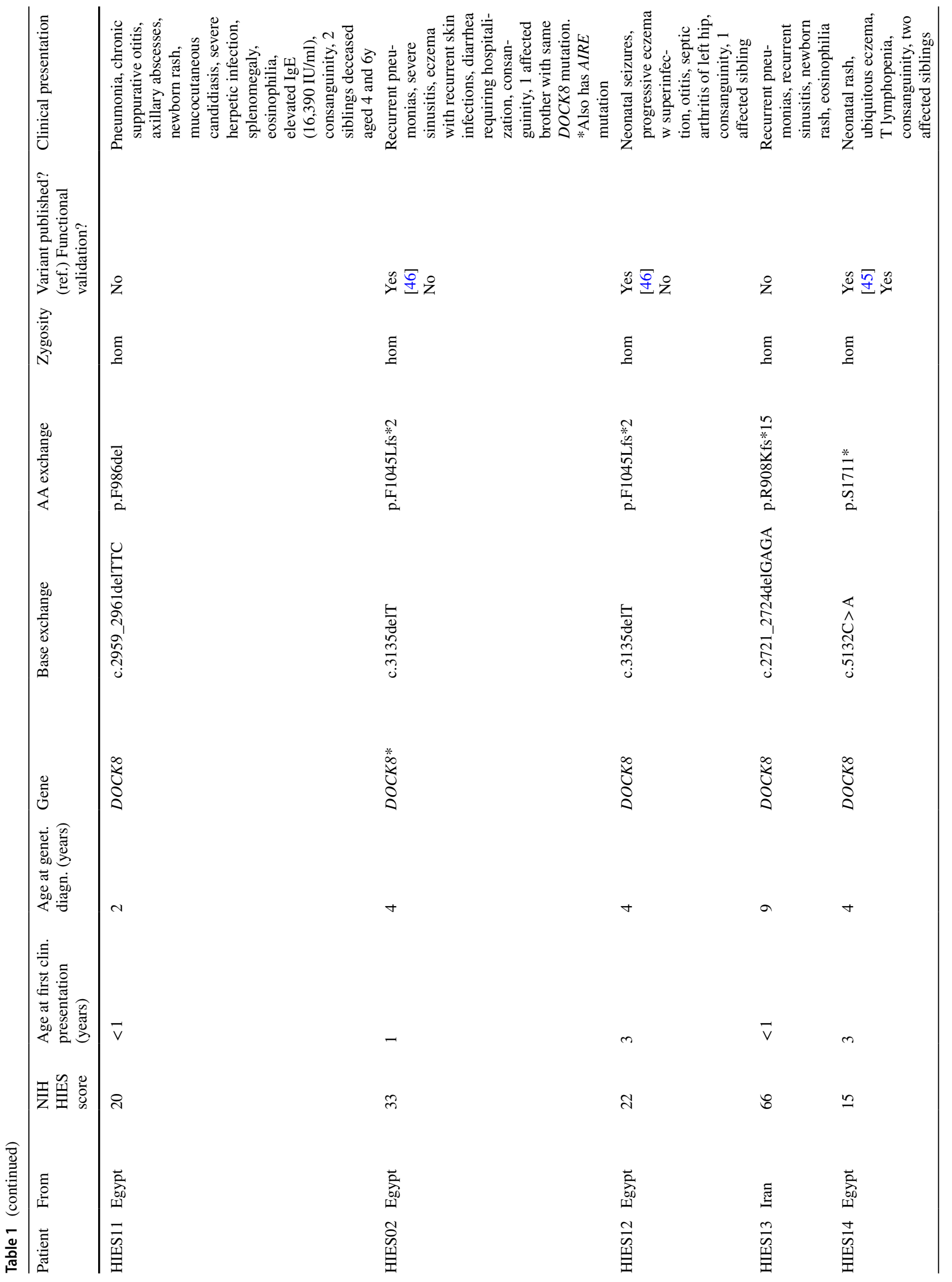




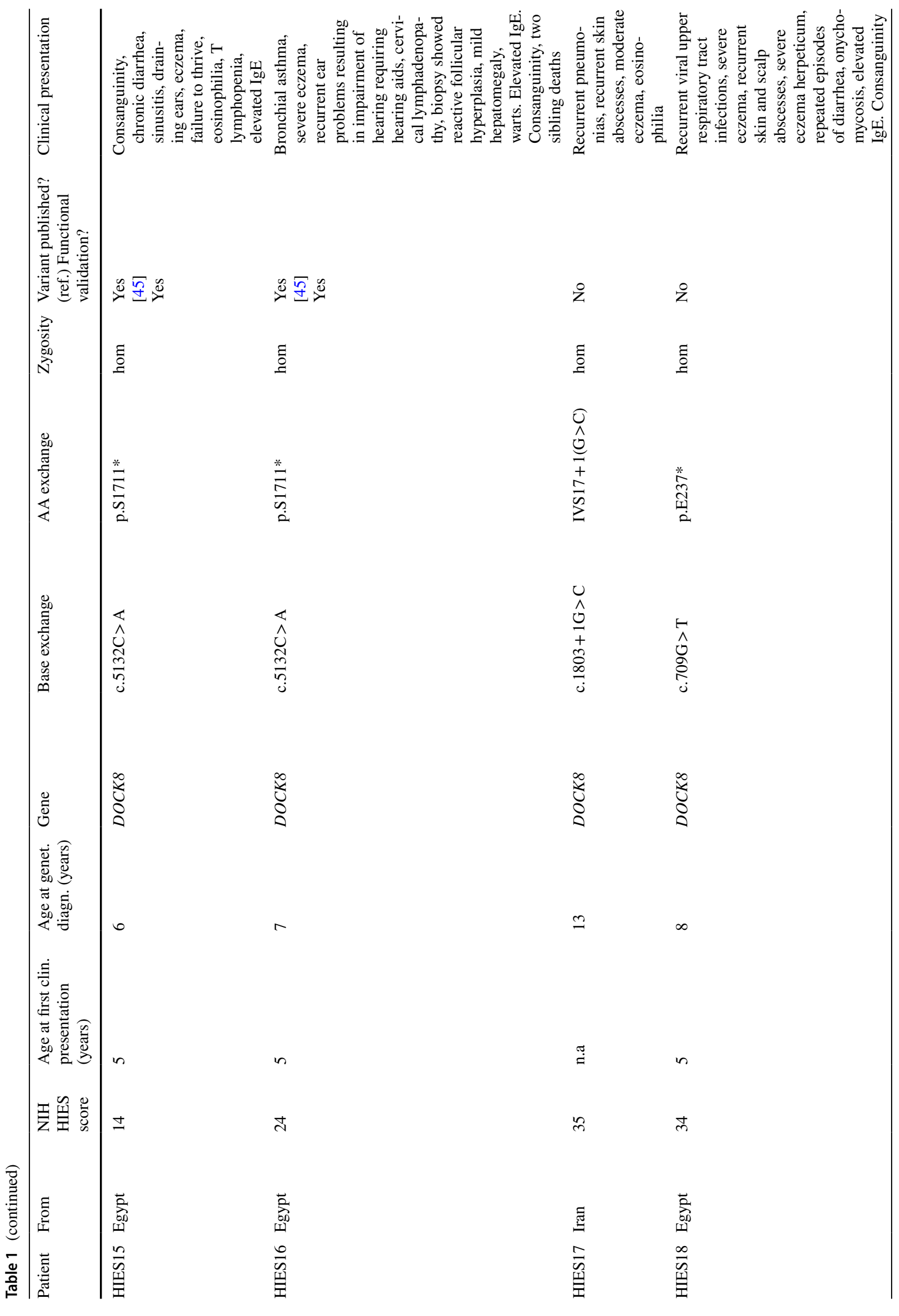




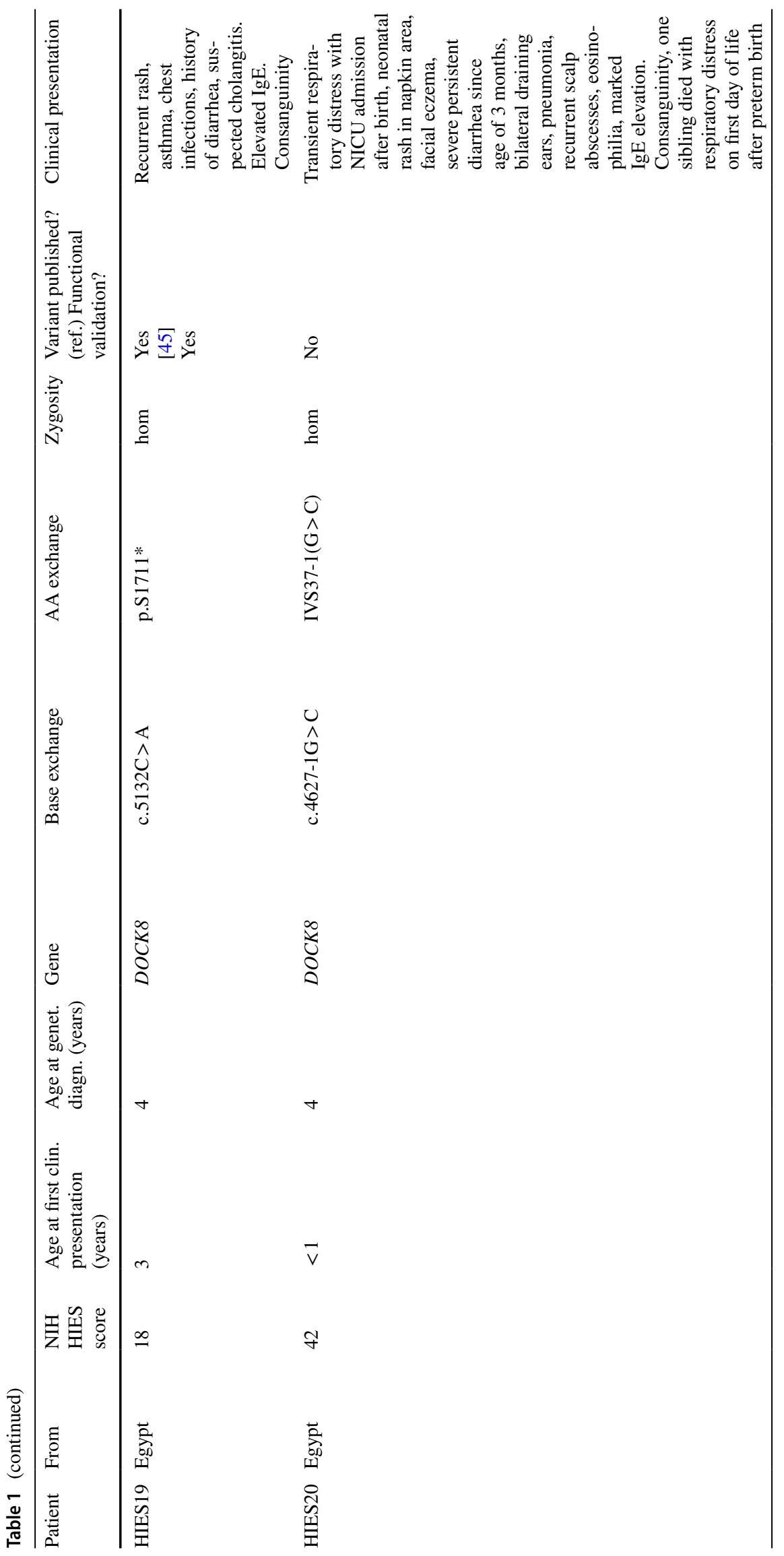




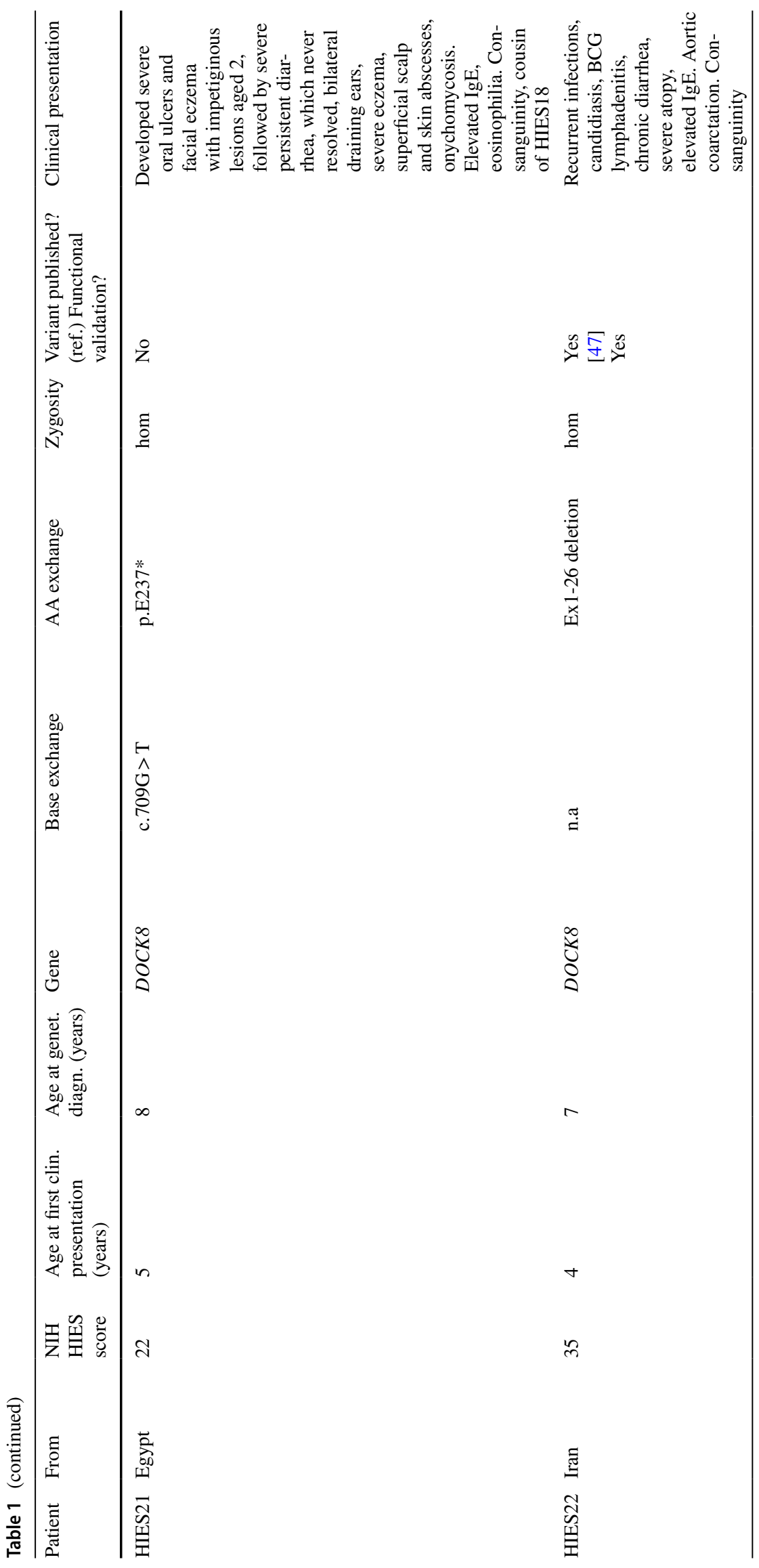




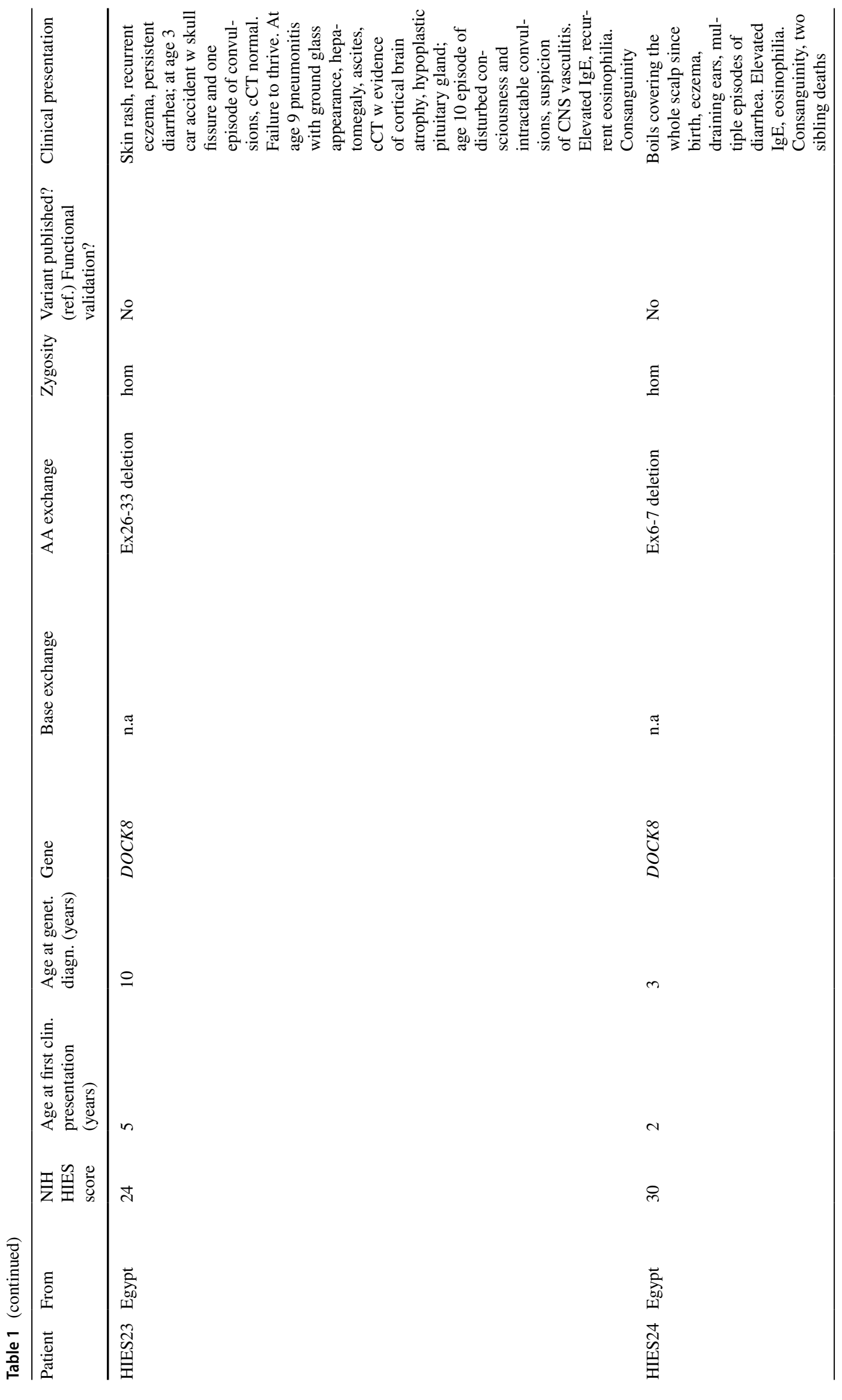




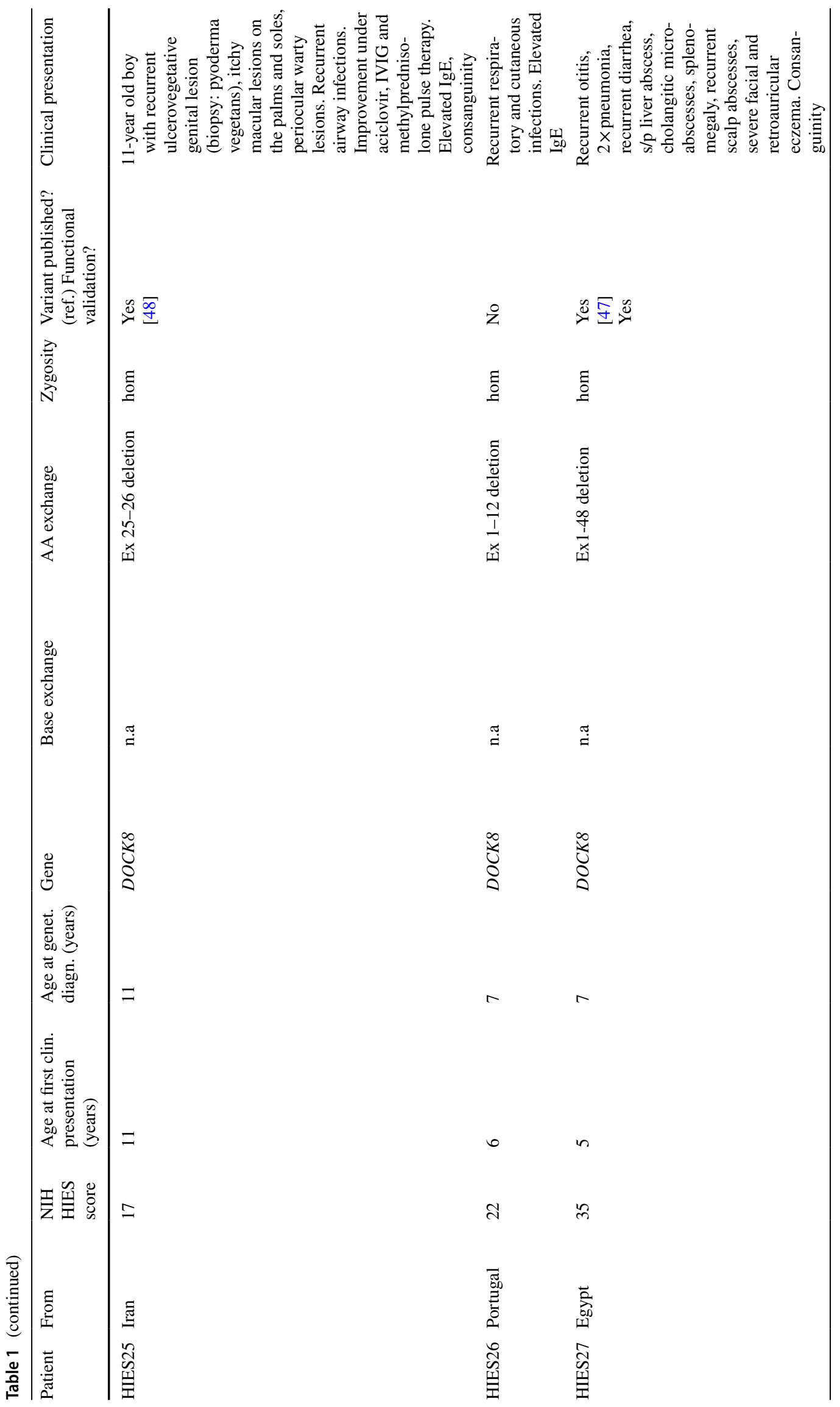




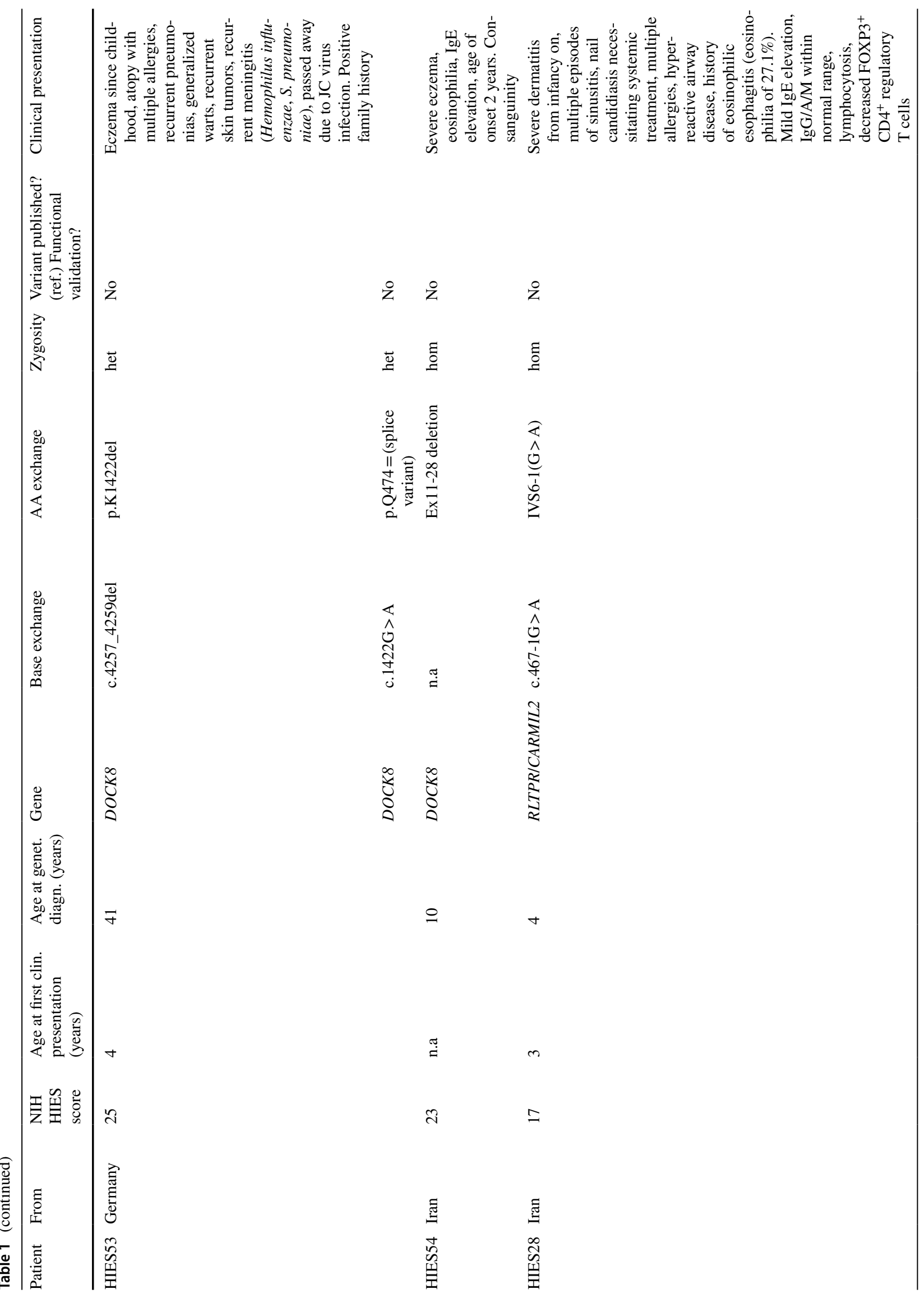




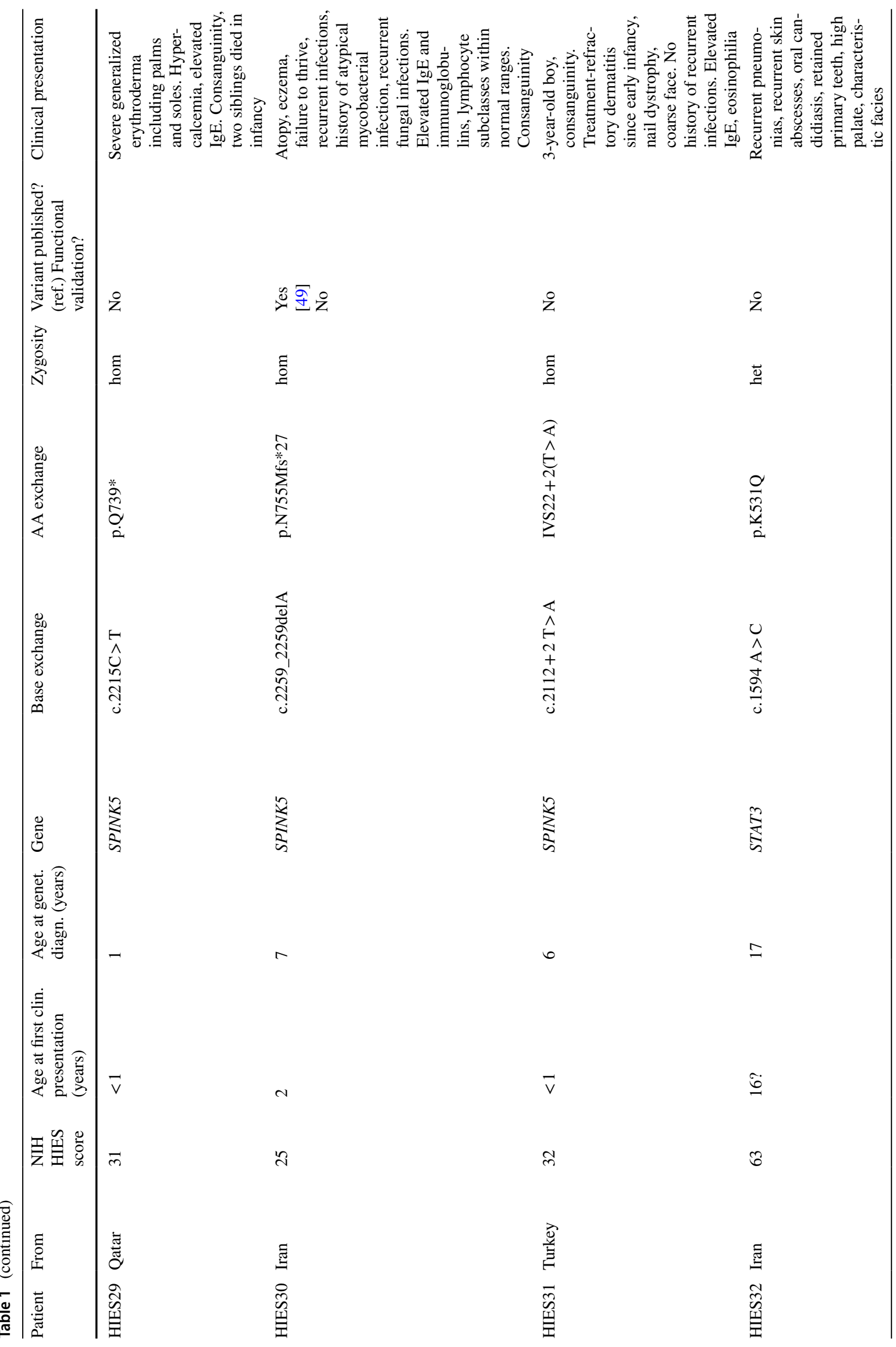




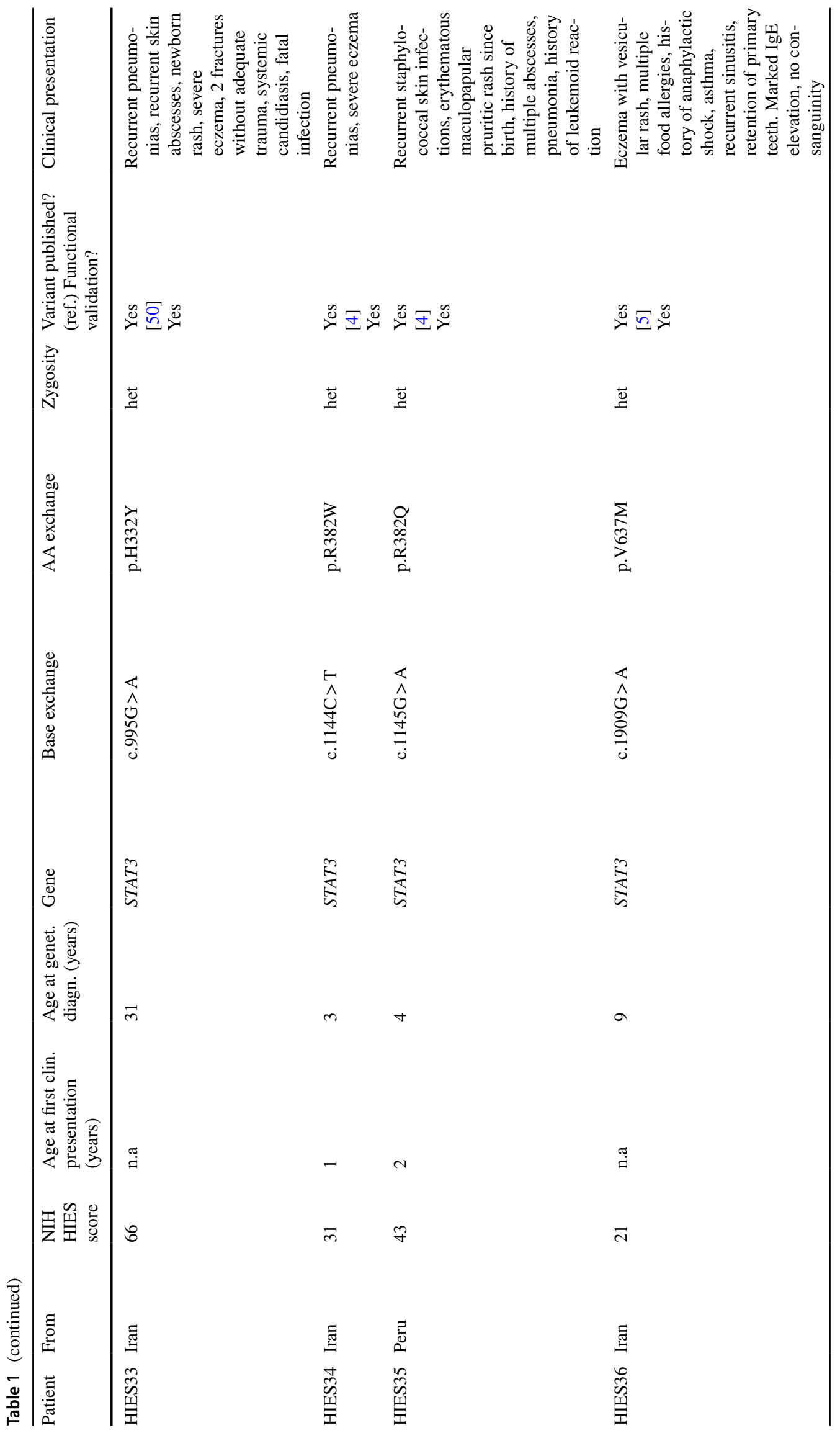




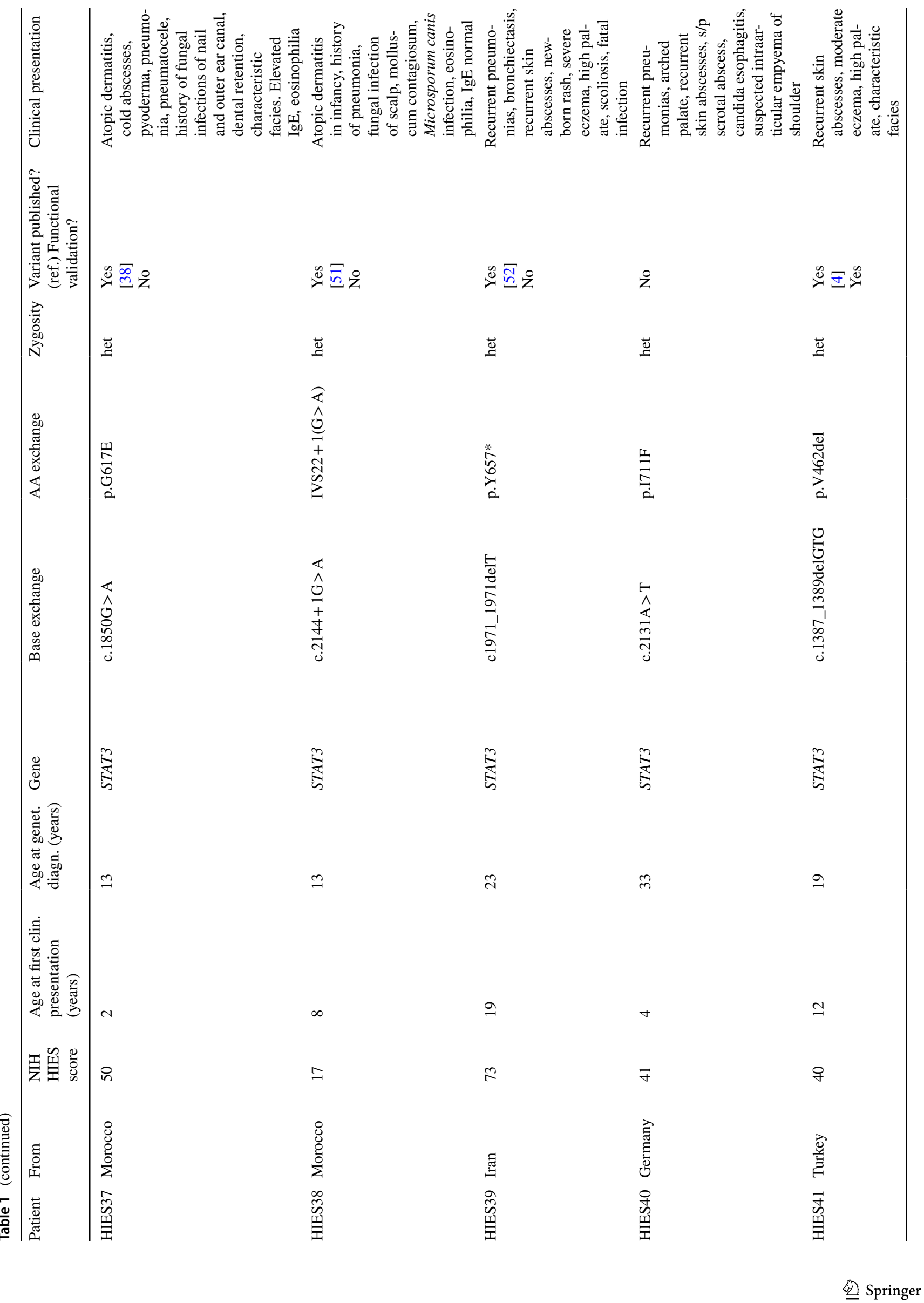




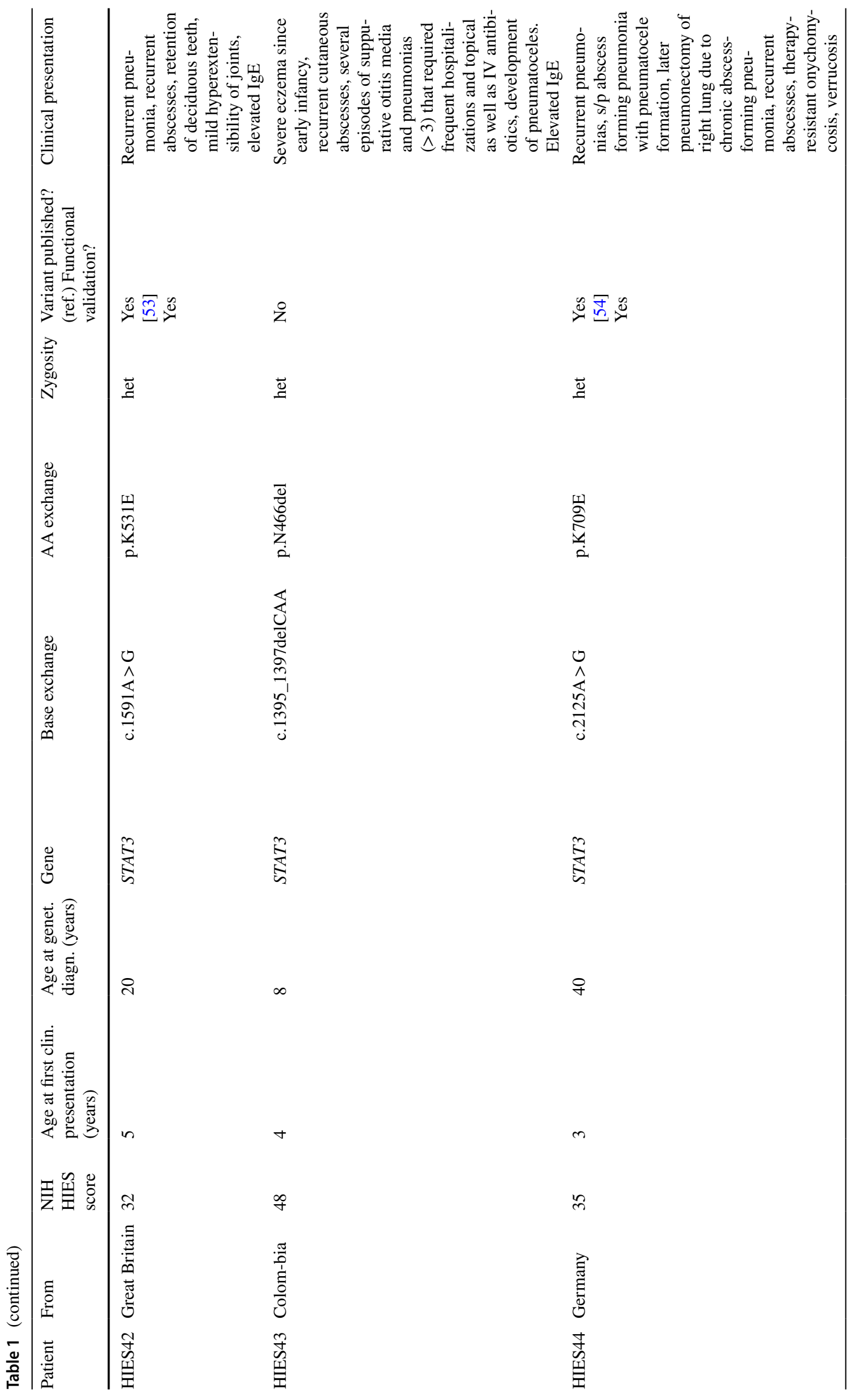




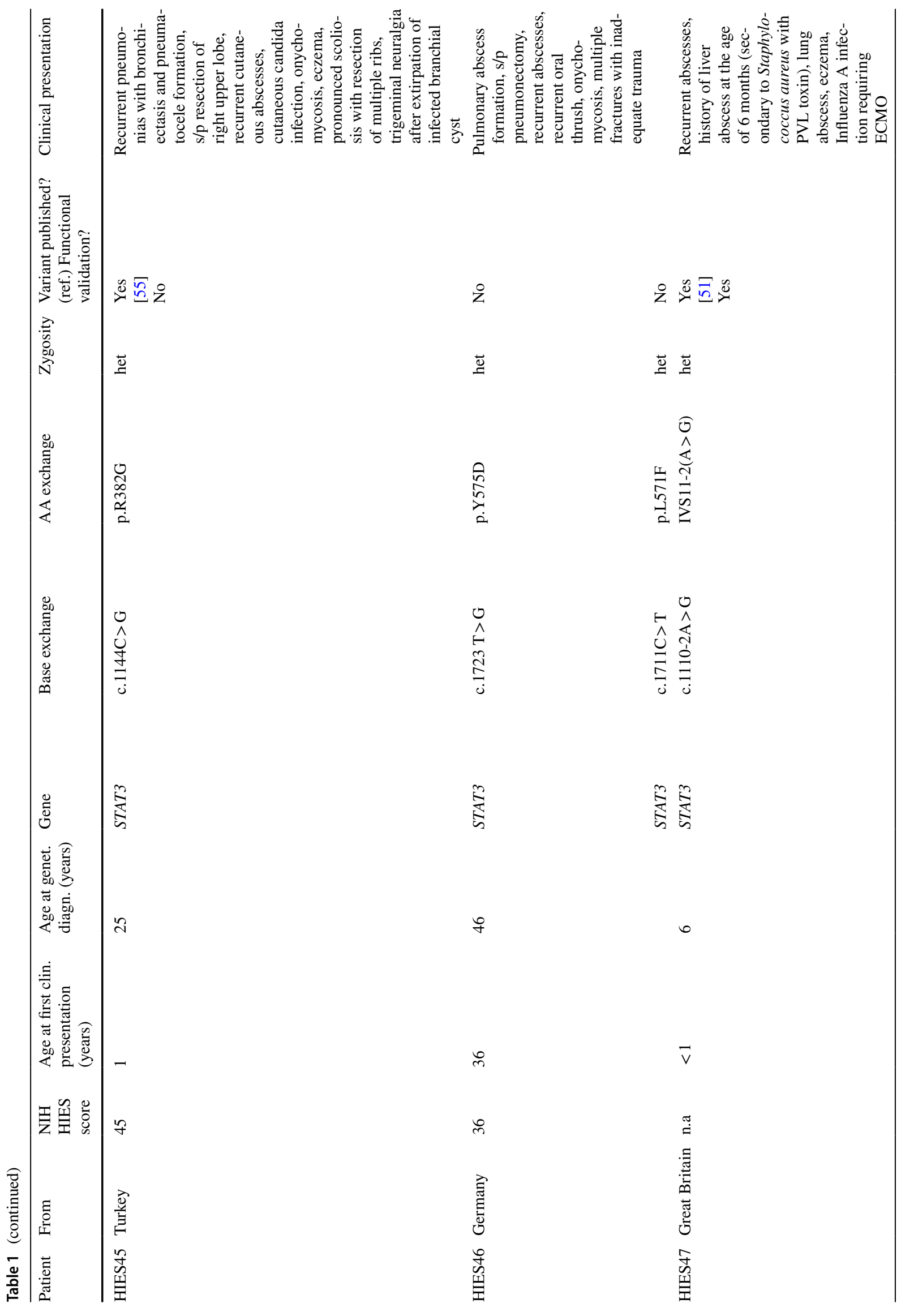




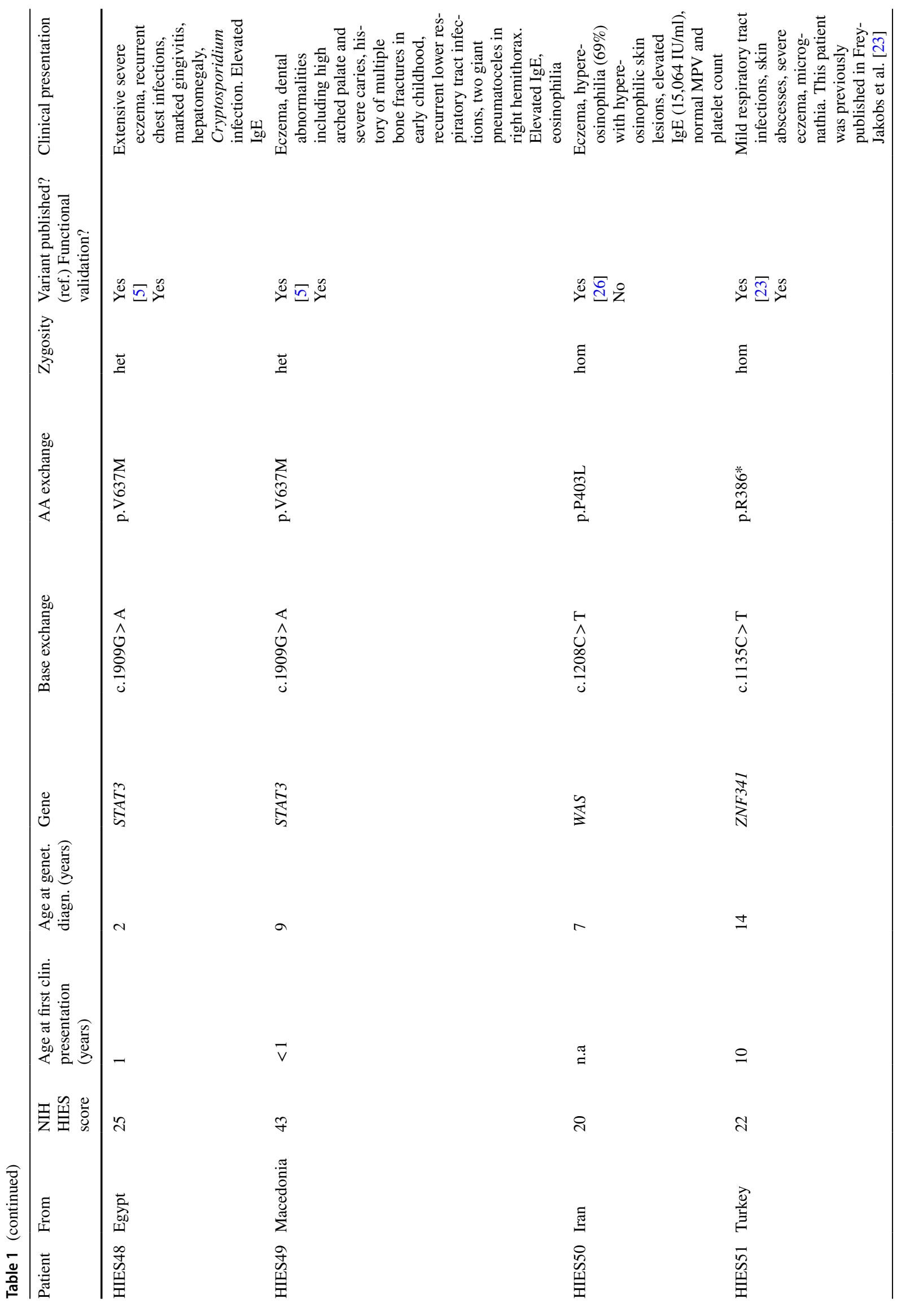




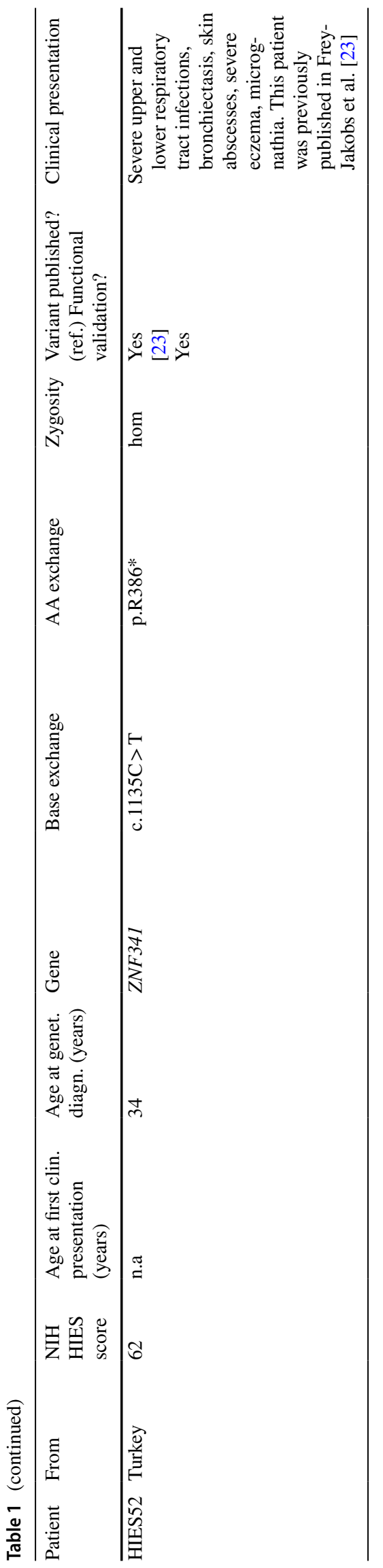

thrombocytopenia [26]. The patient had eczema, marked hypereosinophilia $(69 \%$, absolute count: $21,962 / \mu \mathrm{l})$ with hypereosinophilic skin lesions, and elevated IgE levels $(15,064 \mathrm{IU} / \mathrm{ml})$. Platelet count and platelet volume were noted to be normal.

Additionally, we have identified one patient with a homozygous splice site defect in RLTPR, who had been clinically diagnosed with HIES. The patient suffered from severe dermatitis from infancy on and, in addition, had multiple episodes of sinusitis and nail candidiasis necessitating systemic treatment as well as hyperreactive airway disease. He has multiple allergies and was diagnosed with eosinophilic esophagitis at the age of 2 years (eosinophilia of 27.1\%). The clinical phenotype is thus similar to the one published by Alazami et al. [27], although this patient so far only demonstrated mild signs of immunodeficiency compared to the combined immunodeficiency phenotype described originally by Wang et al. [28]. Recurrent measurements showed mildly elevated $\operatorname{IgE}$, but other immunoglobulins and vaccination responses were normal. Further laboratory workup showed persistent lymphocytosis. The patient was shown to have decreased $\mathrm{FOXP} 3{ }^{+} \mathrm{CD} 4^{+}$regulatory $\mathrm{T}$ cells in line with the so far described phenotype.

Furthermore, two patients had compound heterozygous mutations in AIRE. Patient HIES01 suffered from asthma, sinusitis, and mild eczema and was found to have a multinodular goiter and a large pulmonary cyst. Laboratory workup showed elevated IgE levels. Additionally, he had a Marfanoid habitus and osteoporosis with a history of multiple fractures upon minor trauma, leading to the clinical diagnosis of HIES. The other patient (HIES02) additionally had a homozygous frameshift mutation of DOCK 8 and thus had presented with recurrent pneumonias, severe sinusitis, and eczema with recurrent skin infections and diarrhea.

\section{Identified Genetic Defects in Patients with a Clinical Diagnosis of CMC}

Out of 64 patients with a clinical diagnosis of CMC, targeted panel sequencing allowed us to make a genetic diagnosis in 24 patients, i.e., $37.5 \%$. A detailed list of detected mutations and clinical presentations of patients can be found in Table 2. As expected, gain-of-function mutations in STAT1 were the most common identified defects (Fig. 4). Specifically, 15 CMC patients were shown to carry heterozygous missense mutations in STAT1; some of these patients have been previously published [22, 29]. Out of these, one patient additionally had a compound heterozygous $C A R D 9$ mutation and another one a mutation in ILI7RA.

Four CMC patients were found to have homozygous or compound heterozygous mutations in CARD9. One patient had a homozygous nonsense mutation, two had homozygous missense mutations, and one patient had a compound 


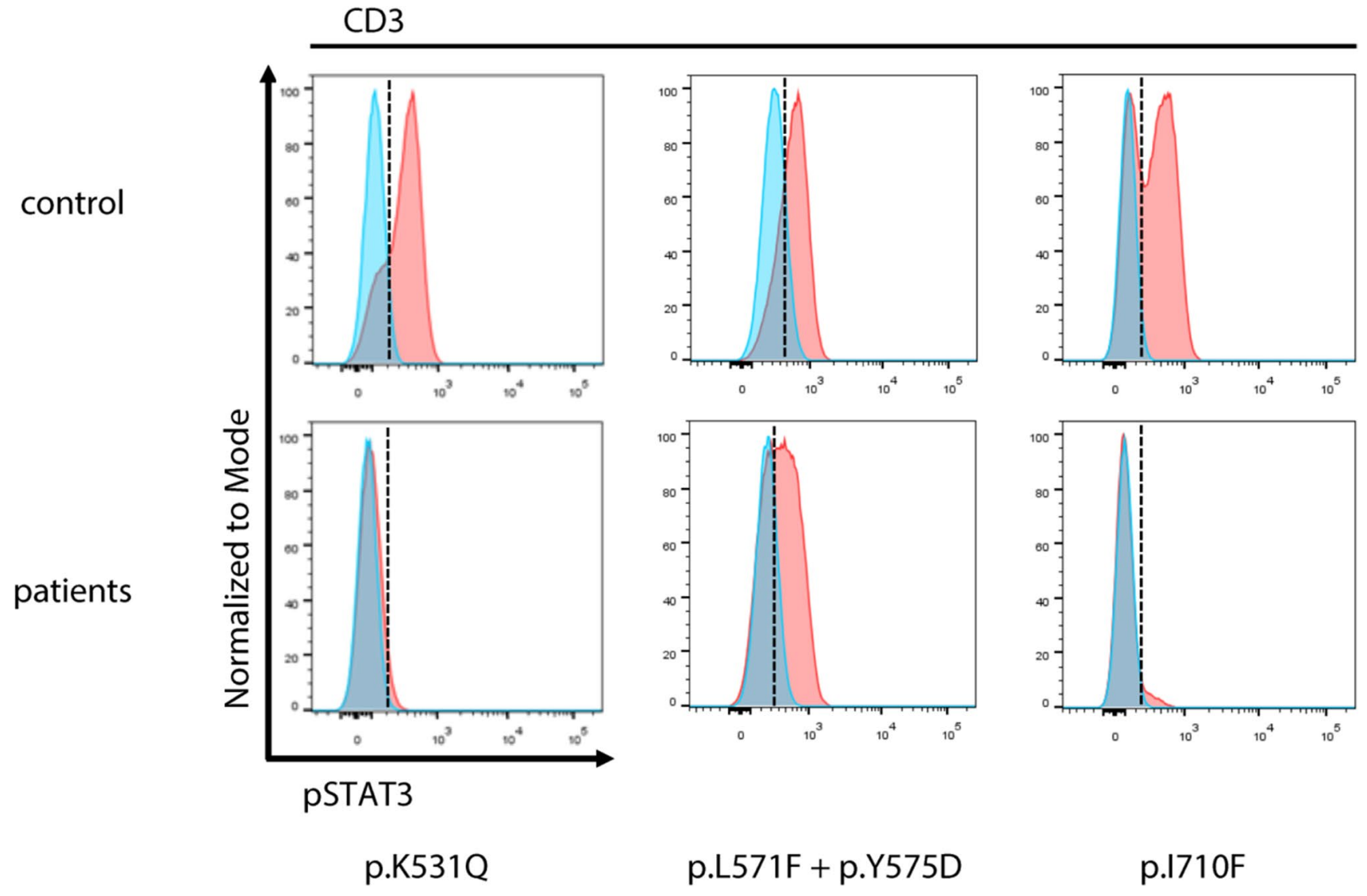

Fig. 3 Functional assessment of novel STAT3 defects: flow cytometric analysis of pSTAT3 in $\mathrm{CD}^{+}$cells of patients shows hypophosphorylation of STAT3 upon stimulation with IL-6 for the assessed

heterozygous mutation consisting of a heterozygous frameshift mutation and a heterozygous splice site mutation. Two of these four patients had suffered from candida meningoencephalitis.

Furthermore, two patients were found to have mutations in ILI7RA. One patient had a homozygous frameshift mutation, where flow cytometric analysis showed severely reduced surface expression of ILI7RA (see Fig. 5). The other patient had a heterozygous nonsense mutation (p.R66*) and a heterozygous missense variant predicted to be damaging (p.W320R). Primary cells of this patient for staining were not available.

One patient was shown to have a heterozygous missense variant in AIRE (p.R471C), which has previously been published as being associated with autoimmune features but not overt APECED (autoimmune-polyendocrinopathy-candidiasis-ectodermal dystrophy). He had suffered from recurrent oral candida infections in infancy and was reported to have recurrent respiratory tract infections and obstructive bronchitis. Anticytokine autoantibodies against IL17 or IL22, which are commonly observed in APECED, were below the detection level. However, the patient was shown to have variants, p.K531Q and p.I710F, and to a lesser extent, p.L571F/p. Y575D. While not diagnostic, this result suggests pathogenicity for the assessed variants

autoantibodies against IL28A/B, which have previously been described in APECED, but do not belong to the most prevalent anticytokine autoantibodies [30]. The type III interferons IL28A/B have been shown to play a role in antifungal immunity [31], thus these autoantibodies may play a role in the development of CMC.

Finally, three patients with the clinical diagnosis of CMC had heterozygous mutations located in the linker domain of STAT3 (p.F561del and p.D570N). Functional workup of the F561del mutation had shown normal STAT3 expression and functional phosphorylation of STAT1 and STAT3. Further analysis showed reduced STAT3 target gene activation and increased STATl target gene activation, thus mimicking a STATl gain-of-function phenotype [32] with a clinical presentation of mucocutaneous candidiasis.

\section{Identified Genetic Defects in the Previously Sanger-Sequenced Subcohort}

Out of 90 patients, in whom previous Sanger sequencing PCR amplification of the suspected underlying target gene (STAT3, DOCK8, PGM3, STAT1) had not led to a definite 


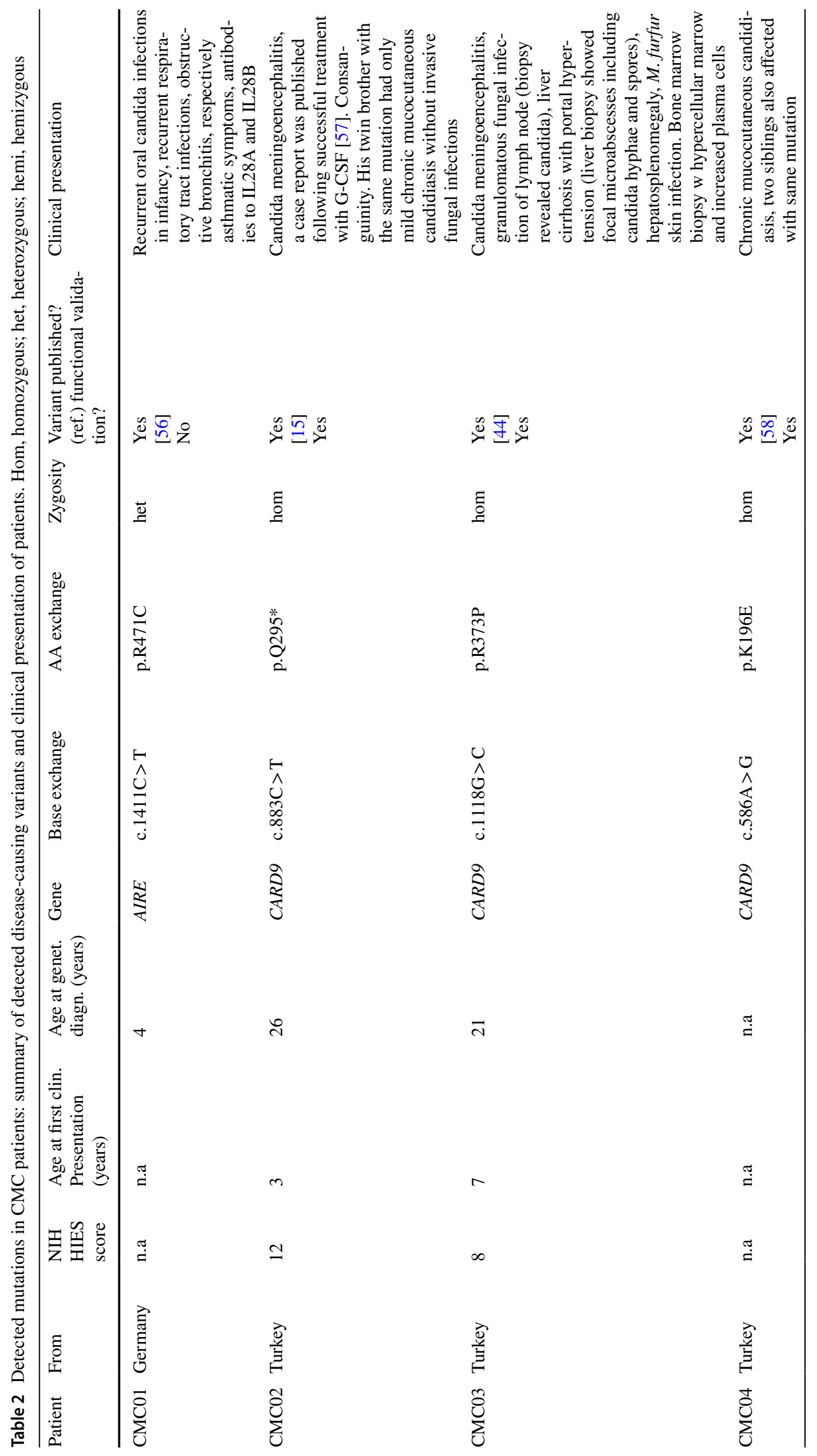




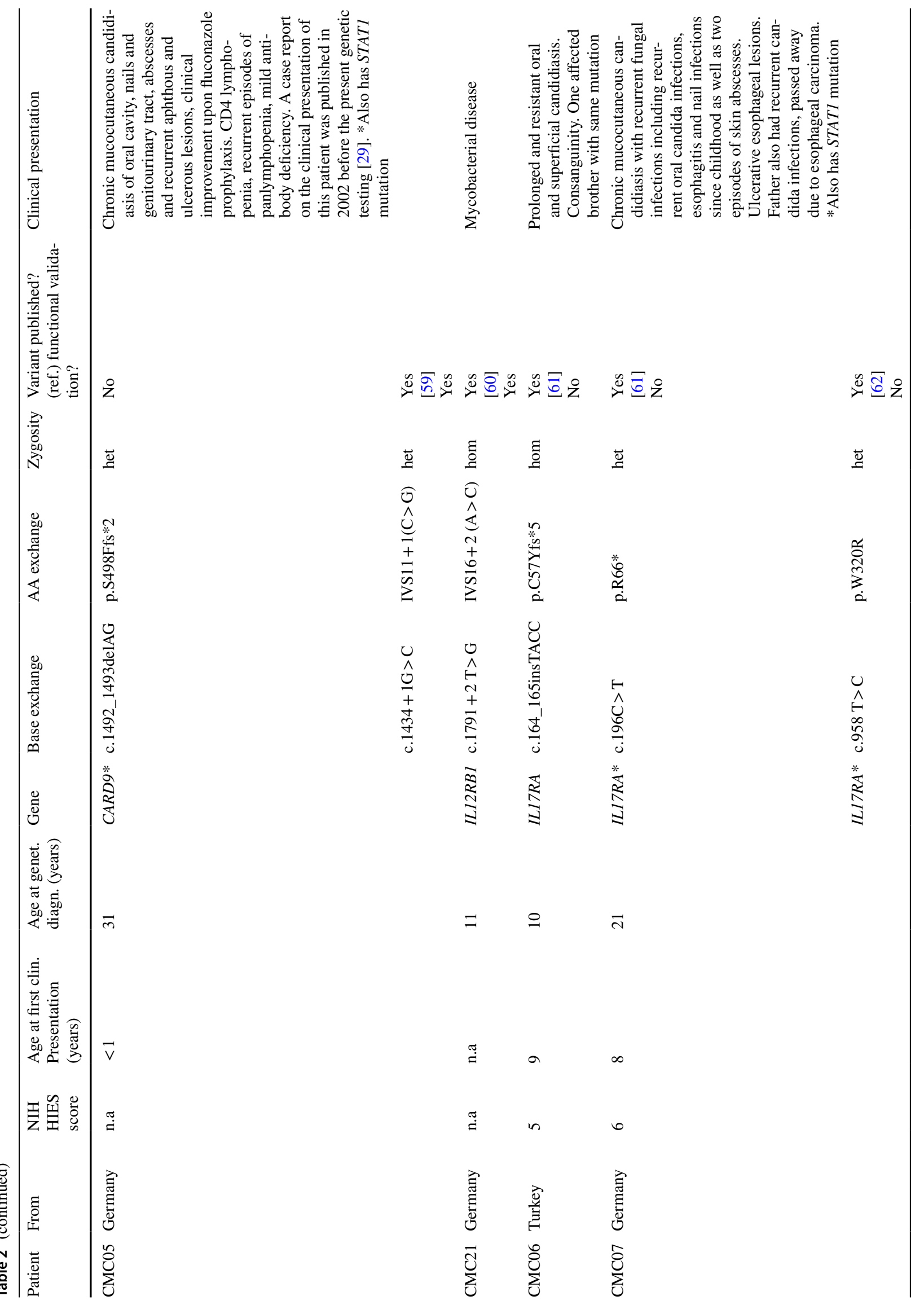




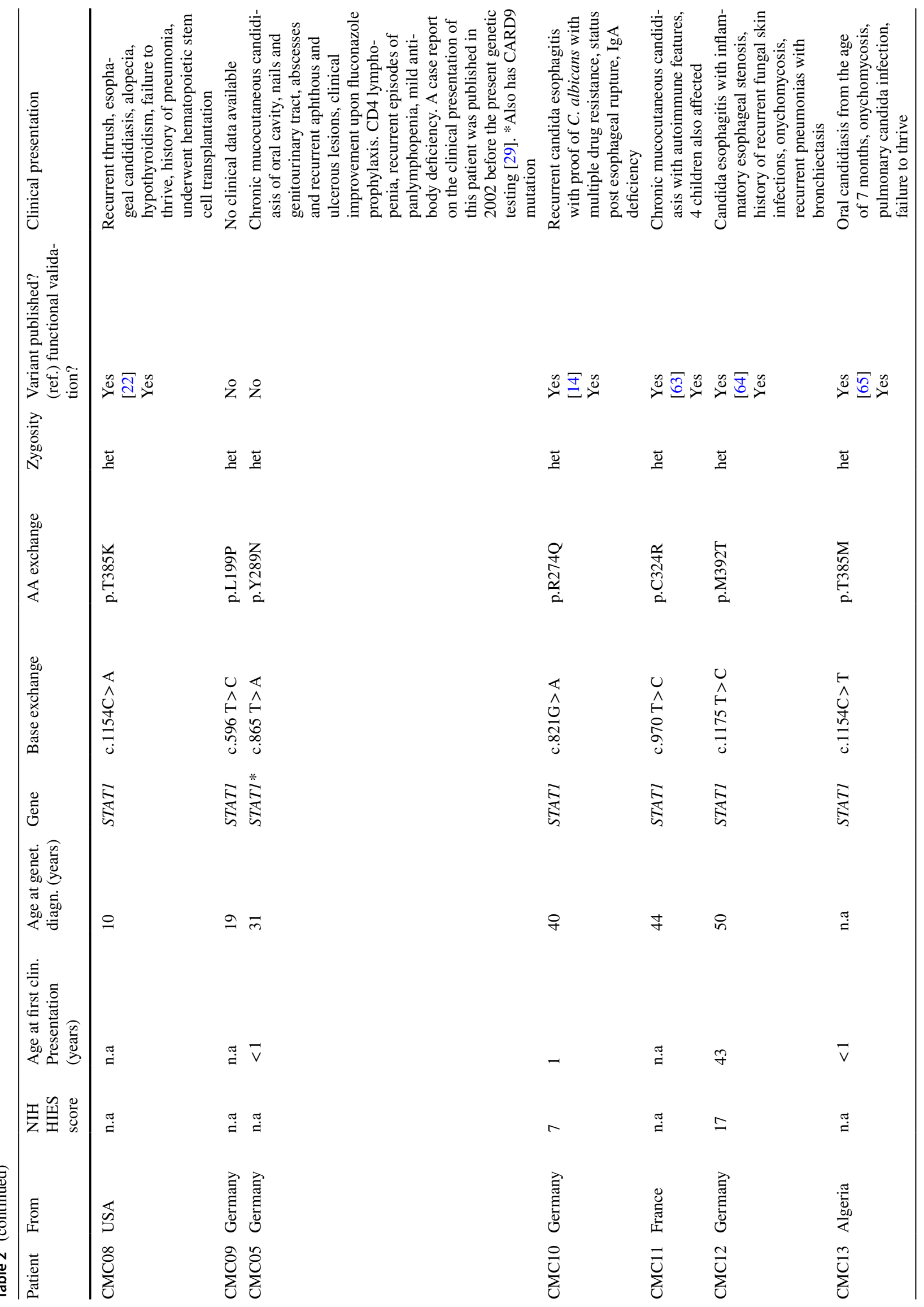




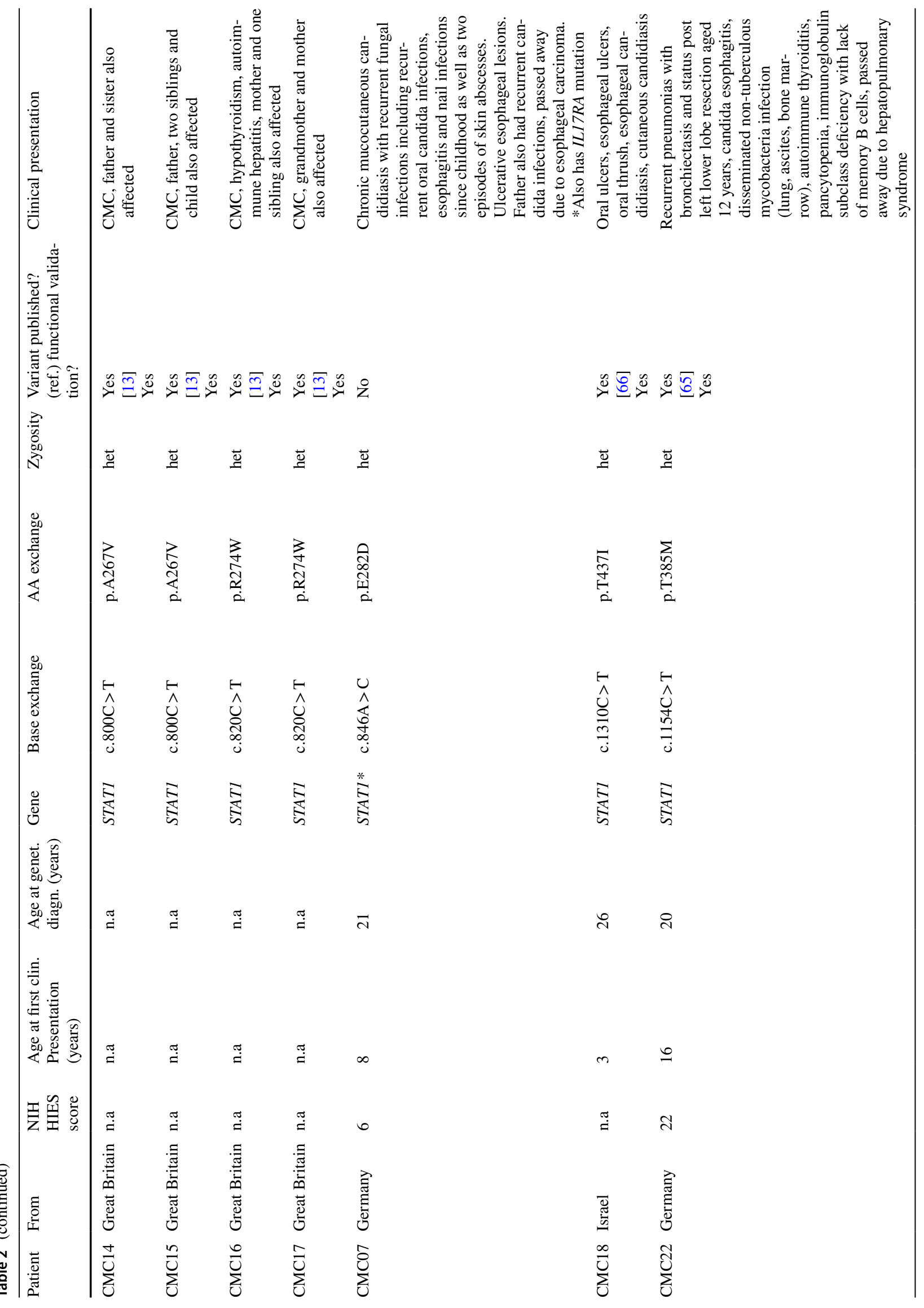




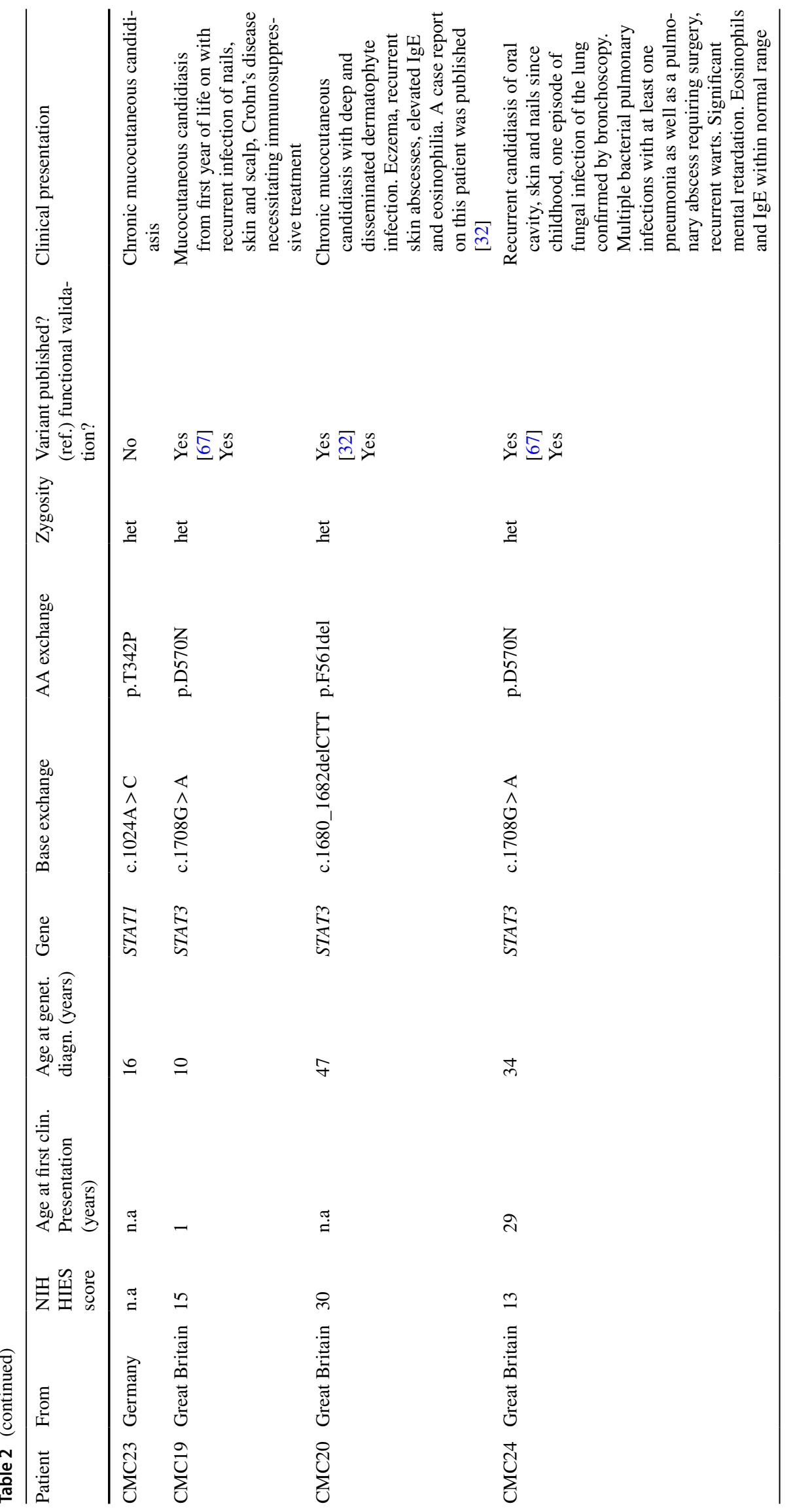




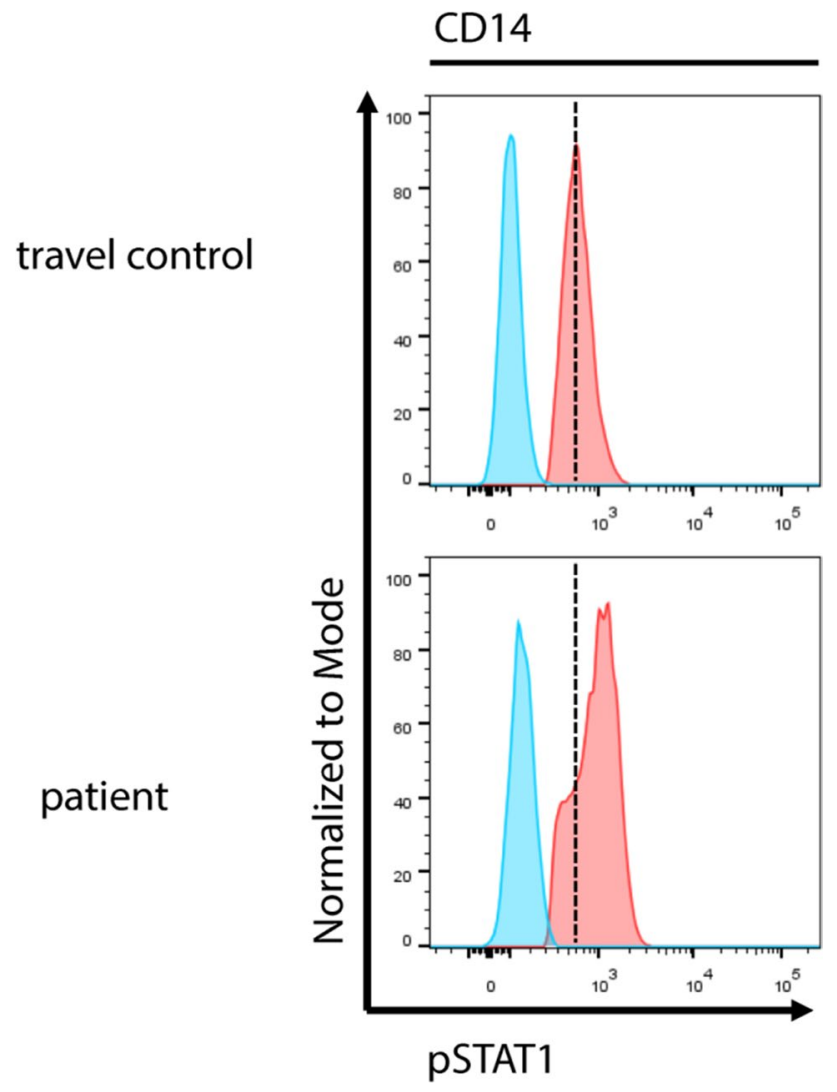

Fig. 4 Functional assessment of novel STAT1 variant p.E282D: flow cytometric analysis of pSTAT1 in $\mathrm{CD}_{14}{ }^{+}$cells of patient $\mathrm{CMC} 07$ demonstrates that the novel STAT1 variant p.E282D leads to hyperphosphorylation of STAT1 upon stimulation with IFN $\alpha$, confirming a gain-of-function mutation

molecular diagnosis, we were only able to detect a genetic defect in 13 patients, i.e., $14.4 \%$ by panel sequencing. Here, the most common defects identified by panel sequencing were STAT3 mutations (7 patients), followed by homozygous DOCK 8 variants (3 patients). One patient each had a homozygous or compound heterozygous mutation in CARD9, ZNF341, and AIRE. Out of the seven patients with STAT3 mutations, three had previously undergone Sanger sequencing for STAT3. This suggests that although Sanger sequencing was considered the gold standard for genetic testing, this method is not free of errors itself and in the case of strong clinical suspicion, further genetic testing should be considered.

\section{Discussion}

In this study, we used a targeted gene panel sequencing approach to search for underlying mutations in a cohort of 211 patients clinically diagnosed with HIES and 64 patients diagnosed with CMC by their referring physicians. In total,

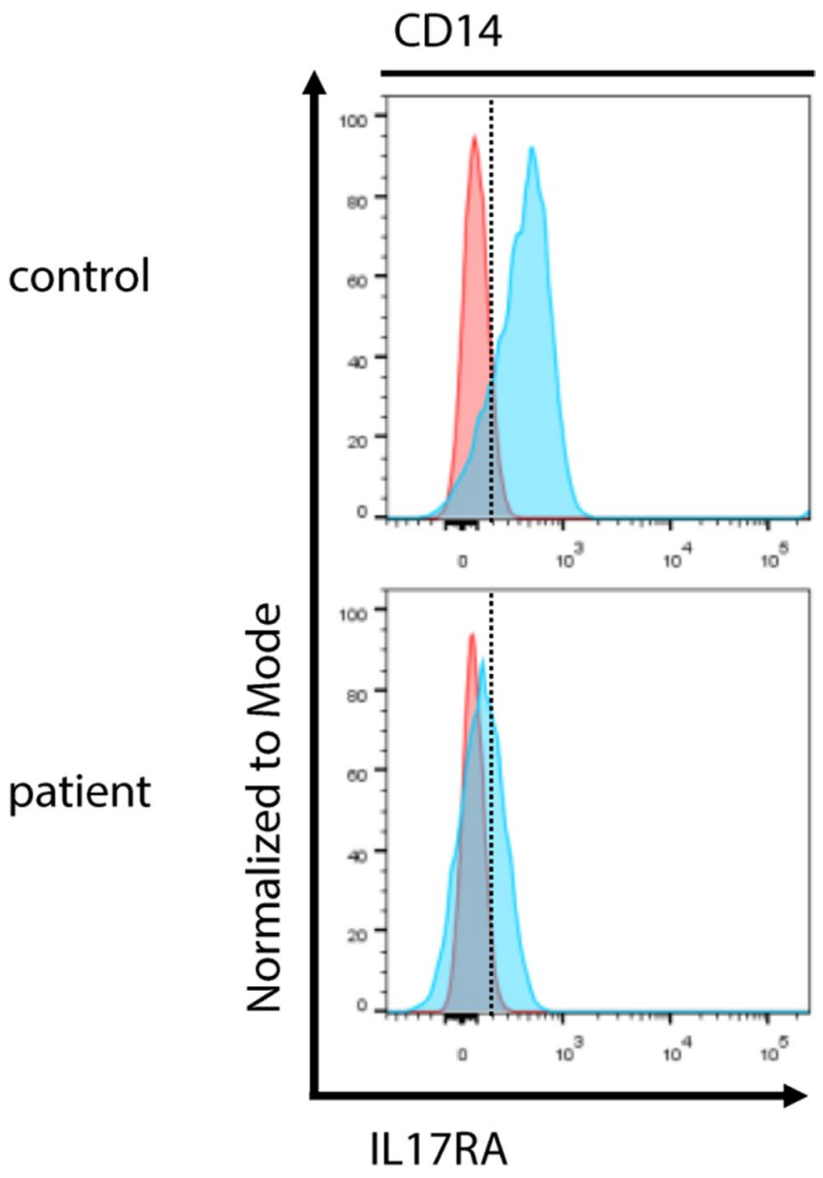

Fig. 5 Assessment of IL17RA expression: flow cytometric analysis of IL17RA in $\mathrm{CD}_{14}{ }^{+}$cells shows reduced surface expression in patient CMC06 with the homozygous frameshift variant p.C57Yfs*5

we have detected 87 mutations in 78 patients. This translates into a diagnostic hit rate of $28.4 \%$ in our cohort, which is in line with other studies published on next-generation sequencing approaches in primary immunodeficiencies, which have reported rates of 15 to $40 \%$ [33-36], depending on patient preselection. Out of the 87 detected mutations, 32 constituted novel mutations, whereas 55 mutations had been published before.

Due to many patients within this cohort hailing from Middle Eastern countries, such as Egypt, Turkey, Iran, and Morocco, where consanguinity is more common, there was a selection bias, which may account for the fact that DOCK 8 mutations were the most commonly identified defects in this cohort. Previous reports have identified STAT defects as the most common defects in European cohorts [37]. Similarly, among patients with European ancestry, also in this cohort, STAT1 and STAT3 defects were by far the most prevalent. Furthermore, we cannot rule out that previous Sanger sequencing of a part of the cohort may have led to further bias. Out of an overall cohort of 613 HIES and 93 CMC patients analyzed by our laboratory, 322 had undergone 
Sanger sequencing for the target gene deemed most likely, revealing 91 STAT3, 39 DOCK8, and 5 PGM3 mutations in HIES and 23 STAT1 and 4 CARD 9 mutations in the CMC cohort. Thus, overall, STAT3 and STAT1 constituted the most frequent defects.

While it was originally published that $T Y K 2$ mutations may cause a hyper-IgE phenotype, this phenotype could not be replicated and hence $T Y K 2$ has been removed from the list of HIES defects in the IUIS classification. Also, in our cohort, we did not detect any mutations in $T Y K 2$, confirming that an HIES phenotype is not typically caused by mutations in $T Y K 2$.

The three most commonly reported clinical features in the described cohort were IgE elevation, eczema, and eosinophilia, which are common to all hyper-IgE phenotypes but are unfortunately largely unspecific. The most common condition with this phenotype is severe atopic dermatitis [38]. Several authors have addressed the issue of how to distinguish primary immunodeficiency from atopic disease [38-40]. Schimke et al. found internal abscesses and severe infections among other features predictive of primary immunodeficiency (PID) [38]. In this cohort, $8.2 \%$ of patients had no history of major or increased minor infections, whereas $41.7 \%$ did not have a history of severe infections (pneumonia or other severe or fatal infection), rendering the diagnosis of immunodeficiency uncertain. Thus, despite their clinical diagnosis of HIES, some patients may in fact suffer from other conditions within the spectrum of hyper-IgE phenotypes. This hypothesis is supported by the fact that we have detected variants in filaggrin $(F L G)$ in patients from this cohort, some of which are known to be associated with ichthyosis vulgaris and atopic dermatitis.

In addition, individual noninfectious features were nonspecific to the mutational status. A characteristic facies was the most commonly reported skeletal feature in this cohort (23.1\%); however, this is not an objectively quantifiable criterion and relies heavily on the expertise of the referring physician. Bone fractures with inadequate trauma were reported in six patients with STAT3 mutations (6/21) but also in six patients without detected genetic defect (6/197). Severe scoliosis was reported in two patients with STAT3 defects (2/21) and seven patients without detected genetic defects (7/197). Pneumatocele formation was reported in five patients (5/21) with STAT3 mutations, one (1/3) with AIRE mutation, and eight (8/197) without any detected genetic defect. This is consistent with earlier findings from Schimke et al. describing pneumatoceles, nail or mucocutaneous candidiasis, bone fractures without adequate trauma, and scoliosis to be predictive of STAT3. While, thus, distinguishing STAT3 from other genetic defects, these features fail to clearly distinguish patients with and without a genetic defect. These findings highlight again that each individual clinical feature by itself is nonspecific. For this reason, scores such as the HIES NIH score were developed to predict underlying genetic defects with a higher likelihood. However, these are only available for the more common and established hyperIgE phenotypes such as STAT3 and DOCK8, and an overall score to our knowledge so far does not exist. Furthermore, scoring systems such as the NIH HIES score may impede an early diagnosis as they rely on morphological criteria that develop late in childhood (e.g., teeth, skeletal anomalies) and on the manifestation of infections and end-organ damage, which may take years to become obvious. This issue may be circumvented by early genetic analysis for underlying defects. Broader sequencing may help particularly to identify atypical presentations.

In this study, the targeted panel sequencing approach has allowed us to identify mutations in patients with atypical clinical presentations, such as CARD9 mutations in patients with a clinical diagnosis of the hyper-IgE syndrome, STAT3 mutations in patients with chronic mucocutaneous candidiasis and patients with low HIES scores that may otherwise have been missed, or a WAS mutation in a patient with a typical HIES phenotype.

This highlights the importance of making a genetic diagnosis, as the clinical picture may be variable and the genetic background unexpected. However, the latter may have crucial relevance regarding the counseling and treatment of the patient and their family members. Furthermore, several patients with mutations in multiple genes were identified, which may contribute to atypical, complex, or more severe phenotypes. In addition, the in-depth molecular characterization of underlying mutations and the involved pathways constitutes a prerequisite for the identification of novel therapeutic targets and the development of targeted drug therapy and novel therapeutic approaches such as gene surgery.

In our experience, the targeted panel sequencing approach provided a cost-effective first-line genetic screening method. Material costs for panel sequencing amounted to $€ 141$ per sample, whereas commercial whole exome sequencing costs $€ 600-€ 700$ including data processing for a single patient and approximately $€ 1400$ for a trio of child and parents. Data analysis for panel sequencing does not require complex bioinformatical analysis but can be performed on preexisting software, rendering analysis time and cost effective.

Covering a smaller number of genes highly preselected for those known to be disease causing reduces the effort and time spent on interpreting variants of unclear significance. While panel sequencing precludes the identification of novel genetic defects, the process is more time efficient. Particularly, in a diagnostic setting, fast turnaround times are crucial to ensure a quick diagnosis, thus favoring a panel sequencing approach.

The average coverage of detected mutations in this study amounted to 914 reads. The achieved high coverage is one of the distinct advantages of panel sequencing over the whole 
exome and especially whole genome sequencing and is crucial to reduce errors [41, 42], particularly in a diagnostic setting. Disadvantages to panel sequencing include the constant need to update the panel to reflect the current literature and the need for further follow-up, including further sequencing in case no variants are detected. This is also a limitation of this study as the majority of patients without detection of a genetic defect were not subjected to further genetic workup. The variable number of genes sequenced due to the continuous adaptation of the panel may be regarded as a further limitation of this study.

For the time being, however, we propose a stepwise approach with targeted panel sequencing as the first step in genetic diagnostics. For patients, in whom no mutation could be detected, further workup, including whole exome sequencing, should be considered especially in the case of young age and positive family history and if further family members are available for sequencing. In the future, whole exome sequencing (WES) will probably substitute panel sequencing in the diagnostic setting as both WES and whole genome sequencing and the ensuing data analysis are becoming cheaper and quicker and databases with annotation of variants are improving. The continued improvement of sequencing technologies and bioinformatics analysis will further facilitate the discovery of single nucleotide variants and indels (insertions/deletions) and other structural rearrangements in the future.

In conclusion, we present a targeted panel sequencing approach for the identification of genetic variants underlying the clinical spectrum of hyper-IgE syndromes and mucocutaneous candidiasis. In total, we have detected 87 mutations in 78 out of 275 patients, of which 32 were novel mutations.

Supplementary Information The online version contains supplementary material available at https://doi.org/10.1007/s10875-021-01086-4.

Author Contribution NF and JRR prepared the draft of the manuscript. $\mathrm{NF}$, JRR, ACG, and MP performed data analysis. JRR, MB, and KH performed experiments. LGD, SS, ZC, PSP, NG, MA, JCAB, MSAD, ÖA, TPA, FBK, FC, HR, SSK, IK, CK, RK, ASK, DL, PL, NM, AM, TTÖ, AAK, SS, RS, ABS, EU, TP, VW, HvB, MW, RD, and ZJ provided patient care, collected samples, collected and provided clinical data, and corrected the manuscript. BG supervised the project, provided resources, and reviewed and edited the manuscript.

Funding Open Access funding enabled and organized by Projekt DEAL. Financial support for this research came from the German Ministry of Education and Research (BMBF, grant no. 01E01303; sysINFLAME grant nos. 01ZX1306F and 01ZX1306A), from the E-rare program of the European Commission EURO-CMC (01GM1502), and from the German Center for Infection Research DZIF (8000805-3 and TTU-IICH_07.801). This study was supported by the German Research Foundation (DFG) under Germany's Excellence Strategy "CIBSS" (EXC-2189) (Project ID 390939984) and "RESIST" (EXC 2155) (Project ID 39087428).
Availability of Data and Materials Data can be made available upon request.

Code Availability Not applicable.

\section{Declarations}

Ethics Approval As mentioned previously, this study was conducted under the ethics protocols 239/99-120,733 and 302/13 of the ethics committee of the University Hospital of Freiburg, Germany.

Consent for Participation/Publication All patients, or for children their legal guardians, were consented according to local ethics guidelines for participation/publication. All authors agree to the publication of this manuscript.

Conflict of Interest The authors declare that the research was conducted in the absence of any commercial or financial relationships that could be construed as a potential conflict of interest. The funding organizations had no role in study design, the collection, analysis and interpretation of data, the writing of the report, or the decision to submit the paper for publication.

Open Access This article is licensed under a Creative Commons Attribution 4.0 International License, which permits use, sharing, adaptation, distribution and reproduction in any medium or format, as long as you give appropriate credit to the original author(s) and the source, provide a link to the Creative Commons licence, and indicate if changes were made. The images or other third party material in this article are included in the article's Creative Commons licence, unless indicated otherwise in a credit line to the material. If material is not included in the article's Creative Commons licence and your intended use is not permitted by statutory regulation or exceeds the permitted use, you will need to obtain permission directly from the copyright holder. To view a copy of this licence, visit http://creativecommons.org/licenses/by/4.0/.

\section{References}

1. Davis SD, Schaller J, Wedgwood RJ. Job's syndrome Recurrent, "cold", staphylococcal abscesses. Lancet. 1966;1(7445):1013-5.

2. Buckley RH, Wray BB, Belmaker EZ. Extreme hyperimmunoglobulinemia $\mathrm{E}$ and undue susceptibility to infection. Pediatrics. 1972;49(1):59-70.

3. Grimbacher B, Holland SM, Gallin JI, Greenberg F, Hill SC, Malech HL, et al. Hyper-IgE syndrome with recurrent infections-an autosomal dominant multisystem disorder. N Engl J Med. 1999;340(9):692-702.

4. Minegishi Y, Saito M, Tsuchiya S, Tsuge I, Takada H, Hara $\mathrm{T}$, et al. Dominant-negative mutations in the DNA-binding domain of STAT3 cause hyper-IgE syndrome. Nature. 2007;448(7157):1058-62.

5. Holland SM, DeLeo FR, Elloumi HZ, Hsu AP, Uzel G, Brodsky $\mathrm{N}$, et al. STAT3 mutations in the hyper-IgE syndrome. N Engl J Med. 2007;357(16):1608-19.

6. Renner ED, Puck JM, Holland SM, Schmitt M, Weiss M, Frosch $\mathrm{M}$, et al. Autosomal recessive hyperimmunoglobulin E syndrome: a distinct disease entity. J Pediatr. 2004;144(1):93-9.

7. Zhang Q, Davis JC, Lamborn IT, Freeman AF, Jing H, Favreau AJ, et al. Combined immunodeficiency associated with DOCK8 mutations. N Engl J Med. 2009;361(21):2046-55. 
8. Minegishi Y, Saito M, Morio T, Watanabe K, Agematsu K, Tsuchiya S, et al. Human tyrosine kinase 2 deficiency reveals its requisite roles in multiple cytokine signals involved in innate and acquired immunity. Immunity. 2006;25(5):745-55.

9. Sassi A, Lazaroski S, Wu G, Haslam SM, Fliegauf M, Mellouli $\mathrm{F}$, et al. Hypomorphic homozygous mutations in phosphoglucomutase 3 (PGM3) impair immunity and increase serum IgE levels. J Allergy Clin Immunol. 2014;133(5):1410-9 (9 e1-13).

10. Felgentreff K, Siepe M, Kotthoff S, von Kodolitsch Y, Schachtrup $\mathrm{K}$, Notarangelo LD, et al. Severe eczema and Hyper-IgE in Loeys-Dietz-syndrome-contribution to new findings of immune dysregulation in connective tissue disorders. Clin Immunol. 2014;150(1):43-50.

11. Stepensky P, Keller B, Buchta M, Kienzler AK, Elpeleg O, Somech R, et al. Deficiency of caspase recruitment domain family, member 11 (CARD11), causes profound combined immunodeficiency in human subjects. J Allergy Clin Immunol. 2013;131(2):477-85.e1.

12. Wells RS, Higgs JM, Macdonald A, Valdimarsson H, Holt PJ. Familial chronic muco-cutaneous candidiasis. J Med Genet. 1972;9(3):302-10.

13. van de Veerdonk FL, Plantinga TS, Hoischen A, Smeekens SP, Joosten LA, Gilissen C, et al. STAT1 mutations in autosomal dominant chronic mucocutaneous candidiasis. N Engl J Med. 2011;365(1):54-61.

14. Liu L, Okada S, Kong XF, Kreins AY, Cypowyj S, Abhyankar A, et al. Gain-of-function human STAT1 mutations impair IL-17 immunity and underlie chronic mucocutaneous candidiasis. J Exp Med. 2011;208(8):1635-48.

15. Glocker EO, Hennigs A, Nabavi M, Schaffer AA, Woellner $\mathrm{C}$, Salzer U, et al. A homozygous CARD9 mutation in a family with susceptibility to fungal infections. $\mathrm{N}$ Engl J Med. 2009;361(18):1727-35.

16. Puel A, Cypowyj S, Bustamante J, Wright JF, Liu L, Lim HK, et al. Chronic mucocutaneous candidiasis in humans with inborn errors of interleukin-17 immunity. Science. 2011;332(6025):65-8.

17. Stenson PD, Ball EV, Mort M, Phillips AD, Shiel JA, Thomas NS, et al. Human Gene Mutation Database (HGMD): 2003 update. Hum Mutat. 2003;21(6):577-81.

18. Lek M, Karczewski KJ, Minikel EV, Samocha KE, Banks E, Fennell T, et al. Analysis of protein-coding genetic variation in 60,706 humans. Nature. 2016;536(7616):285-91.

19. Sherry ST, Ward M, Sirotkin K. dbSNP-database for single nucleotide polymorphisms and other classes of minor genetic variation. Genome Res. 1999;9(8):677-9.

20. Adzhubei IA, Schmidt S, Peshkin L, Ramensky VE, Gerasimova A, Bork $\mathrm{P}$, et al. A method and server for predicting damaging missense mutations. Nat Methods. 2010;7(4):248-9.

21. Kircher M, Witten DM, Jain P, O'Roak BJ, Cooper GM, Shendure J. A general framework for estimating the relative pathogenicity of human genetic variants. Nat Genet. 2014;46(3):310-5.

22. Depner M, Fuchs S, Raabe J, Frede N, Glocker C, Doffinger R, et al. The extended clinical phenotype of 26 patients with chronic mucocutaneous candidiasis due to gain-of-function mutations in STAT1. J Clin Immunol. 2016;36(1):73-84.

23. Frey-Jakobs S, Hartberger JM, Fliegauf M, Bossen C, Wehmeyer ML, Neubauer JC, et al. ZNF341 controls STAT3 expression and thereby immunocompetence. Sci Immunol. 2018;3(24).

24. Béziat V, Li J, Lin JX, Ma CS, Li P, Bousfiha A, et al. A recessive form of hyper-IgE syndrome by disruption of ZNF341-dependent STAT3 transcription and activity. Sci Immunol. 2018;3(24).

25. Grimbacher B, Schäffer AA, Holland SM, Davis J, Gallin JI, Malech HL, et al. Genetic linkage of hyper-IgE syndrome to chromosome 4. Am J Hum Genet. 1999;65(3):735-44.
26. Leinøe E, Zetterberg E, Kinalis S, Østrup O, Kampmann P, Norström E, et al. Application of whole-exome sequencing to direct the specific functional testing and diagnosis of rare inherited bleeding disorders in patients from the Öresund Region. Scandinavia Br J Haematol. 2017;179(2):308-22.

27. Alazami AM, Al-Helale M, Alhissi S, Al-Saud B, Alajlan H, Monies D, et al. Novel CARMIL2 mutations in patients with variable clinical dermatitis, infections, and combined immunodeficiency. Front Immunol. 2018;9:203.

28. Wang Y, Ma CS, Ling Y, Bousfiha A, Camcioglu Y, Jacquot S, et al. Dual T cell- and B cell-intrinsic deficiency in humans with biallelic RLTPR mutations. J Exp Med. 2016;213(11):2413-35.

29. von Bernuth H, Knochel B, Winkler U, Roesler J, Schlesier M, Gahr M. Immunodeficiency with recurrent panlymphocytopenia, impaired maturation of B lymphocytes, impaired interaction of $\mathrm{T}$ and B lymphocytes, and impaired integrity of epithelial tissue: a variant of idiopathic CD4+ T lymphocytopenia? Pediatr Allergy Immunol. 2002;13(5):381-4.

30. Meyer S, Woodward M, Hertel C, Vlaicu P, Haque Y, Kärner J, et al. AIRE-deficient patients harbor unique high-affinity diseaseameliorating autoantibodies. Cell. 2016;166(3):582-95.

31. Espinosa V, Dutta O, McElrath C, Du P, Chang YJ, Cicciarelli B, et al. Type III interferon is a critical regulator of innate antifungal immunity. Sci Immunol. 2017;2(16).

32. Simpson JK, Fröbel P, Seneviratne SL, Brown M, Lowe DM, Grimbacher B, et al. Invasive dermatophyte infection with trichophyton interdigitale is associated with prurigo induced pseudoperforation and a STAT3 mutation. Br J Dermatol. 2017.

33. Moens LN, Falk-Sorqvist E, Asplund AC, Bernatowska E, Smith CI, Nilsson M. Diagnostics of primary immunodeficiency diseases: a sequencing capture approach. PLoS One. 2014;9(12):e114901.

34. Stoddard JL, Niemela JE, Fleisher TA, Rosenzweig SD. Targeted NGS: a cost-effective approach to molecular diagnosis of PIDs. Front Immunol. 2014;5:531.

35. Nijman IJ, van Montfrans JM, Hoogstraat M, Boes ML, van de Corput L, Renner ED, et al. Targeted next-generation sequencing: a novel diagnostic tool for primary immunodeficiencies. J Allergy Clin Immunol. 2014;133(2):529-34.

36. Al-Mousa H, Abouelhoda M, Monies DM, Al-Tassan N, AlGhonaium A, Al-Saud B, et al. Unbiased targeted next-generation sequencing molecular approach for primary immunodeficiency diseases. J Allergy Clin Immunol. 2016;137(6):1780-7.

37. Yong PF, Freeman AF, Engelhardt KR, Holland S, Puck JM, Grimbacher B. An update on the hyper-IgE syndromes. Arthritis Res Ther. 2012;14(6):228.

38. Schimke LF, Sawalle-Belohradsky J, Roesler J, Wollenberg A, Rack A, Borte M, et al. Diagnostic approach to the hyper-IgE syndromes: immunologic and clinical key findings to differentiate hyper-IgE syndromes from atopic dermatitis. J Allergy Clin Immunol. 2010;126(3):611-7.e1.

39. Hagl B, Heinz V, Schlesinger A, Spielberger BD, SawalleBelohradsky J, Senn-Rauh M, et al. Key findings to expedite the diagnosis of hyper-IgE syndromes in infants and young children. Pediatr Allergy Immunol. 2016;27(2):177-84.

40. Ponsford MJ, Klocperk A, Pulvirenti F, Dalm VASH, Milota T, Cinetto F, et al. Hyper-IgE in the allergy clinic-when is it primary immunodeficiency? Allergy. 2018;73(11):2122-36.

41. Bloch-Zupan A, Jamet X, Etard C, Laugel V, Muller J, Geoffroy $\mathrm{V}$, et al. Homozygosity mapping and candidate prioritization identify mutations, missed by whole-exome sequencing, in SMOC2, causing major dental developmental defects. Am J Hum Genet. 2011;89(6):773-81.

42. Xue Y, Ankala A, Wilcox WR, Hegde MR. Solving the molecular diagnostic testing conundrum for Mendelian disorders in the era 
of next-generation sequencing: single-gene, gene panel, or exome/ genome sequencing. Genet Med. 2015;17(6):444-51.

43. Meloni A, Perniola R, Faà V, Corvaglia E, Cao A, Rosatelli MC. Delineation of the molecular defects in the AIRE gene in autoimmune polyendocrinopathy-candidiasis-ectodermal dystrophy patients from Southern Italy. J Clin Endocrinol Metab. 2002;87(2):841-6.

44. Drewniak A, Gazendam RP, Tool AT, van Houdt M, Jansen $\mathrm{MH}$, van Hamme JL, et al. Invasive fungal infection and impaired neutrophil killing in human CARD9 deficiency. Blood. 2013;121(13):2385-92.

45. Bittner TC, Pannicke U, Renner ED, Notheis G, Hoffmann $\mathrm{F}$, Belohradsky BH, et al. Successful long-term correction of autosomal recessive hyper-IgE syndrome due to DOCK8 deficiency by hematopoietic stem cell transplantation. Klin Padiatr. 2010;222(6):351-5.

46. Al-Kzayer LFY, Al-Aradi HMH, Shigemura T, Sano K, Tanaka M, Hamada M, et al. DOCK8 mutation diagnosed using wholeexome sequencing of the dried blood spot-derived DNA: a case report of an Iraqi girl diagnosed in Japan. BMC Med Genet. 2019;20(1):114.

47. Engelhardt KR, McGhee S, Winkler S, Sassi A, Woellner C, Lopez-Herrera $\mathrm{G}$, et al. Large deletions and point mutations involving the dedicator of cytokinesis 8 (DOCK8) in the autosomal-recessive form of hyper-IgE syndrome. J Allergy Clin Immunol. 2009;124(6):1289-302.e4.

48. Engelhardt KR, Gertz ME, Keles S, Schäffer AA, Sigmund EC, Glocker $\mathrm{C}$, et al. The extended clinical phenotype of 64 patients with dedicator of cytokinesis 8 deficiency. J Allergy Clin Immunol. 2015;136(2):402-12.

49. Sheikhbahaei S, Sherkat R, Roos D, Yaran M, Najafi S, Emami A. Gene mutations responsible for primary immunodeficiency disorders: a report from the first primary immunodeficiency biobank in Iran. Allergy Asthma Clin Immunol. 2016;12:62.

50. Jiao H, Tóth B, Erdos M, Fransson I, Rákóczi E, Balogh I, et al. Novel and recurrent STAT3 mutations in hyper-IgE syndrome patients from different ethnic groups. Mol Immunol. 2008;46(1):202-6.

51. Woellner C, Gertz EM, Schaffer AA, Lagos M, Perro M, Glocker EO, et al. Mutations in STAT3 and diagnostic guidelines for hyper-IgE syndrome. J Allergy Clin Immunol. 2010;125(2):424$32(\mathbf{e 8})$.

52. Tavassoli M, Abolhassani H, Yazdani R, Ghadami M, Azizi G, Abdolrahim Poor Heravi S, et al. The first cohort of Iranian patients with hyper immunoglobulin E syndrome: a longterm follow-up and genetic analysis. Pediatr Allergy Immunol. 2019;30(4):469-78.

53. Kim HJ, Kim JH, Shin YK, Lee SI, Ahn KM. A novel mutation in the linker domain of the signal transducer and activator of transcription 3 gene, p.Lys531Glu, in hyper-IgE syndrome. J Allergy Clin Immunol. 2009;123(4):956-8.

54. de Beaucoudrey L, Puel A, Filipe-Santos O, Cobat A, Ghandil $\mathrm{P}$, Chrabieh M, et al. Mutations in STAT3 and IL12RB1 impair the development of human IL-17-producing T cells. J Exp Med. 2008;205(7):1543-50.

55. Kumánovics A, Wittwer CT, Pryor RJ, Augustine NH, Leppert MF, Carey JC, et al. Rapid molecular analysis of the STAT3 gene in Job syndrome of hyper-IgE and recurrent infectious diseases. J Mol Diagn. 2010;12(2):213-9.

56. Cervato S, Morlin L, Albergoni MP, Masiero S, Greggio N, Meossi C, et al. AIRE gene mutations and autoantibodies to interferon omega in patients with chronic hypoparathyroidism without APECED. Clin Endocrinol (Oxf). 2010;73(5):630-6.

57. Celmeli F, Oztoprak N, Turkkahraman D, Seyman D, Mutlu E, Frede N, et al. Successful granulocyte colony-stimulating factor treatment of relapsing Candida albicans meningoencephalitis caused by CARD9 deficiency. Pediatr Infect Dis J. 2016;35(4):428-31.

58. Imanaka Y, Taniguchi M, Doi T, Tsumura M, Nagaoka R, Shimomura $\mathrm{M}$, et al. Inherited CARD9 deficiency in a child with invasive disease due to Exophiala dermatitidis and two older but asymptomatic siblings. J Clin Immunol. 2021.

59. Chiriaco M, Di Matteo G, Conti F, Petricone D, De Luca M, Di Cesare S, et al. First case of patient with two homozygous mutations in. Front Immunol. 2019;10:130.

60. Fieschi C, Dupuis S, Catherinot E, Feinberg J, Bustamante J, Breiman A, et al. Low penetrance, broad resistance, and favorable outcome of interleukin 12 receptor beta1 deficiency: medical and immunological implications. J Exp Med. 2003;197(4):527-35.

61. Lévy R, Okada S, Béziat V, Moriya K, Liu C, Chai LY, et al. Genetic, immunological, and clinical features of patients with bacterial and fungal infections due to inherited IL-17RA deficiency. Proc Natl Acad Sci U S A. 2016;113(51):E8277-85.

62. Besnard V, Calender A, Bouvry D, Pacheco Y, Chapelon-Abric C, Jeny $F$, et al. G908R NOD2 variant in a family with sarcoidosis. Respir Res. 2018;19(1):44.

63. Sharfe N, Nahum A, Newell A, Dadi H, Ngan B, Pereira SL, et al. Fatal combined immunodeficiency associated with heterozygous mutation in STAT1. J Allergy Clin Immunol. 2014;133(3):807-17.

64. Toubiana J, Okada S, Hiller J, Oleastro M, Lagos Gomez M, Aldave Becerra JC, et al. Heterozygous STAT1 gain-of-function mutations underlie an unexpectedly broad clinical phenotype. Blood. 2016;127(25):3154-64.

65. Takezaki S, Yamada M, Kato M, Park MJ, Maruyama K, Yamazaki Y, et al. Chronic mucocutaneous candidiasis caused by a gain-of-function mutation in the STAT1 DNA-binding domain. J Immunol. 2012;189(3):1521-6.

66. Wang X, Zhang R, Wu W, Wang A, Wan Z, van de Veerdonk FL, et al. New and recurrent STAT1 mutations in seven Chinese patients with chronic mucocutaneous candidiasis. Int J Dermatol. 2017;56(2):e30-3.

67. Jägle S, Heeg M, Grün S, Rensing-Ehl A, Maccari ME, Klemann C, et al. Distinct molecular response patterns of activating STAT3 mutations associate with penetrance of lymphoproliferation and autoimmunity. Clin Immunol. 2020;210:108316.

Publisher's Note Springer Nature remains neutral with regard to jurisdictional claims in published maps and institutional affiliations. 


\title{
Authors and Affiliations
}

\author{
Natalie Frede ${ }^{1,2}$. Jessica Rojas-Restrepo ${ }^{1,3}$. Andrés Caballero Garcia de Oteyza ${ }^{1,3}$ - Mary Buchta ${ }^{1}$. \\ Katrin Hübscher ${ }^{1,3}$. Laura Gámez-Díaz ${ }^{1,3}$. Michele Proietti ${ }^{1,3}$. Shiva Saghafi ${ }^{4}$. Zahra Chavoshzadeh ${ }^{5}$. \\ Pere Soler-Palacin ${ }^{6} \cdot$ Nermeen Galal $^{7} \cdot$ Mehdi Adeli $^{8}$. Juan Carlos Aldave-Becerra ${ }^{9} \cdot$ Moudjahed Saleh Al-Ddafari $^{10}$. \\ Ömür Ardenyz ${ }^{11} \cdot$ T. Prescott Atkinson ${ }^{12} \cdot$ Fulya Bektas Kut $^{13} \cdot$ Fatih Çelmeli $^{14} \cdot$ Helen Rees $^{15} \cdot$ Sara S. Kilic $^{16}$. \\ Ilija Kirovski ${ }^{17}$. Christoph Klein ${ }^{18}$ • Robin Kobbe ${ }^{19}$ • Anne-Sophie Korganow ${ }^{20}$ • Desa Lilic ${ }^{21}$ • Peter Lunt ${ }^{22}$. \\ Niten Makwana ${ }^{23}$. Ayse Metin ${ }^{24} \cdot$ Tuba Turul Özgür $^{25}$. Ayse Akman Karakas ${ }^{26}$. Suranjith Seneviratne ${ }^{27}$. \\ Roya Sherkat ${ }^{28}$. Ana Berta Sousa ${ }^{29}$. Ekrem Unal ${ }^{30,31}$. Turkan Patiroglu ${ }^{32} \cdot$ Volker Wahn $^{33}$. \\ Horst von Bernuth ${ }^{33,34,35,36} \cdot$ Margo Whiteford $^{37} \cdot$ Rainer Doffinger ${ }^{38} \cdot$ Zineb Jouhadi $^{39} \cdot$ Bodo Grimbacher 1,3,40,41,42,43
}

1 Center for Chronic Immunodeficiency, Medical Center, Faculty of Medicine, University of Freiburg, Freiburg, Germany

2 Department of Rheumatology and Clinical Immunology, Medical Center, Faculty of Medicine, University of Freiburg, Freiburg, Germany

3 Institute for Immunodeficiency, Medical Center, Faculty of Medicine, University of Freiburg, Freiburg, Germany

4 Immunology Asthma and Allergy Research Institute Tehran University of Medical Sciences, Tehran, Iran

5 Pediatric Infectious Research Center, Mofid Children Hospital, Shahid Beheshti University of Medical Sciences, Tehran, Iran

6 Pediatric Infectious Diseases and Immunodeficiencies Unit, Hospital Universitari Vall D’Hebron, Barcelona, Catalonia, Spain

7 Department of Pediatrics, Faculty of Medicine, Cairo University, Cairo, Egypt

8 Sidra Medicine, Weill Cornell Medicine, Hamad Medical Corporation, Doha, Qatar

$9 \quad$ Allergy and Immunology Division, Hospital Nacional Edgardo Rebagliati Martins, Lima, Peru

10 Laboratory of Applied Molecular Biology and Immunology, University of Abou-Bekr Belkaïd, Tlemcen, Algeria

11 Division of Allergy and Immunology, Department of Internal Medicine, Faculty of Medicine, Ege University, Izmir, Turkey

12 Division of Pediatric Allergy \& Immunology, University of Alabama At Birmingham, Birmingham, AL, USA

13 Departmant of Pediatrics, Division of Pediatric Immunology and Allergy, Faculty of Medicine, Erciyes University, Kayseri, Turkey

14 Antalya Education and Research Hospital Department of Pediatric Immunology and Allergy, Antalya, Turkey

15 Bristol Royal Hospital for Children, University Hospitals Bristol NHS Foundation Trust, Bristol, UK

16 Faculty of Medicine, Uludag University, Bursa, Turkey

17 Medical Faculty Skopje, 50 Divizija BB, 1000 Skopje, Macedonia

18 Department of Pediatrics, Dr. Von Hauner Children's Hospital, University Hospital, LMU Munich, Munich, Germany

19 First Department of Medicine, Division of Infectious Diseases, University Medical Center , Hamburg-Eppendorf, Germany
20 UFR Médecine, Université de Strasbourg, Strasbourg, France

21 Institute of Cellular Medicine, Newcastle University, Newcastle-upon-Tyne, UK

22 Centre for Academic Child Health, University of Bristol, Bristol, UK

23 Department of Pediatrics, Sandwell and West, Birmingham Hospitals NHS Trust, Birmingham, UK

24 Department of Pediatric Allergy and Immunology, Ankara Children's Hematology Oncology Training and Research Hospital, Ankara, Turkey

25 Department of Pediatrics, Division of Immunology, Akdeniz University Medical Faculty, Antalya, Turkey

26 Department of Dermatology and Venerology, Akdeniz University Medical Faculty, Antalya, Turkey

27 Institute of Immunity and Transplantation, Royal Free Hospital and University College London, London, UK

28 Acquired Immunodeficiency Research Center, Isfahan University of Medical Sciences, Isfahan, Iran

29 Serviço de Genética, Hospital de Santa Maria, Centro Hospitalar Universitário Lisboa Norte, and Laboratório de Imunologia Básica, Faculdade de Medicina de Lisboa, Universidade de Lisboa, Lisboa, Portugal

30 Department of Pediatrics, Division of Pediatric Hematology and Oncology, Faculty of Medicine, Erciyes University, 38010, Melikgazi, Kayseri, Turkey

31 Deparment of Molecular Biology and Genetics, Gevher Nesibe Genom and Stem Cell Institution, GENKOK Genome and Stem Cell Center, Erciyes University, 38010, Melikgazi, Kayseri, Turkey

32 Department of Pediatrics, Division of Pediatric Immunology, Faculty of Medicine, Erciyes University, 38010, Melikgazi, Kayseri, Turkey

33 Department of Pediatric Respiratory Medicine, Immunology and Critical Care Medicine, Charité - Universitätsmedizin Berlin, Freie Universität Berlin, Humboldt-Universität $\mathrm{Zu}$ Berlin, and Berlin Institute of Health, Berlin, Germany

34 Department of Immunology, Labor Berlin GmbH, Berlin, Germany

35 Charité - Universitätsmedizin Berlin, corporate member of Freie Universität Berlin, Humboldt-Universität Zu Berlin, Berlin Institute of Health (BIH), Berlin-Brandenburg Center for Regenerative Therapies (BCRT), Berlin, Germany

36 Berlin Institute of Health (BIH), Berlin, Germany 
37 Department of Clinical Genetics, Queen Elizabeth University Hospital, Glasgow G51 4TF, UK

38 Department of Clinical Biochemistry and Immunology, Addenbrooke's Hospital, Cambridge, UK

39 Department of Pediatric Infectious Diseases, Children's Hospital CHU Ibn Rochd, University Hassan 2, Casablanca, Morocco

40 German Center for Infection Research (DZIF), Satellite Center Freiburg, Freiburg, Germany
41 CIBSS - Centre for Integrative Biological Signalling Studies, Albert-Ludwigs University, Freiburg, Germany

42 RESIST - Cluster of Excellence 2155 to Hanover Medical School, Satellite Center Freiburg, Freiburg, Germany

43 CCI-Center for Chronic Immunodeficiency, Universitätsklinikum Freiburg, Breisacher Straße 115, 79106 Freiburg, Germany 